\title{
Characteristic and Expression of Hsp70 and Hsp90 Genes from Tyrophagus Putrescentiae and Their Response to Thermal Stress
}

\author{
Jing Wang \\ Nanchang University \\ Sheng-quan Que \\ Jiangxi Academy of Forestry \\ Meng-ru Jin \\ Nanchang University \\ Xin-yu Liu \\ Nanchang University \\ Tian-rong Xin \\ Nanchang University \\ Zhi-wen Zou \\ Nanchang University \\ Bin Xia ( $\nabla$ xiabin9@163.com ) \\ Nanchang University
}

\section{Research Article}

Keywords: Tyrophagus putrescentiae, Hsp70, Hsp90, gene cloning, RT-qPCR

Posted Date: April 8th, 2021

DOl: https://doi.org/10.21203/rs.3.rs-394909/v1

License: (c) (i) This work is licensed under a Creative Commons Attribution 4.0 International License.

Read Full License 
$1 \quad$ Characteristic and expression of $H s p 70$ and $H s p 90$ genes from

2 Tyrophagus putrescentiae and their response to thermal stress

3

4 Jing Wang ${ }^{1 \&}$, Sheng-quan Que ${ }^{2 \&}$, Mengru Jin ${ }^{1}$, Xinyu Liu ${ }^{1}$, Tian-Rong Xin ${ }^{1}$, Zhi-wen 5

6

7

8

9

${ }^{1}$ School of Life Sciences, Nanchang University, Nanchang 330031, China

${ }^{2}$ Institute of Forest Pest, Jiangxi Academy of Forestry, Nanchang 330013, China

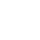

\& These authors contributed equally to this work and should be considered co-first authors,

* Corresponding author at School of Life Sciences, Nanchang University,

Nanchang 330031, China; Tel. and Fax: +86-0791 83968957; Email-address:

451641824@qq.com; xiabin9@163.com

(15)

6.

(1)

8

9




\section{Abstract}

Tyrophagus putrescentiae (T. putrescentiae) is a worldwide tored grain pest mite which has do great harm to human's economy and health. Temperature has a great influence on the population dynamics of $T$. putrescentiae. In this study, the cDNA of $T$. putrescentiae HSP70 and HSP90 (designated TpHSP70-1, TpHSP70-2 and TpHSP90) were cloned, and their expression were determined by fluorescent real time quantitative PCR. Results indicate that TpHSP70-1, TpHSP70-2 and TpHSP9O contained an open reading frame of 1,998 bp, 1,984 bp and 2,157 bp that encoded 665, 661 and 718 amino acid residues, respectively. Sequences and phylogenetic analyses suggested that TpHSP7O and TpHSP90 showed high homology with other species. The RT-qPCR results indicated the relative expression level of TpHSP9O and TpHSP70 gene at different stages were different. Also thermal stress could increase its expression significantly. This study indicates that TpHSP7O and TpHSP9O are very important genes for T. putrescentiae to defend against thermal stress.

Key words: Tyrophagus putrescentiae, Hsp70, Hsp90, gene cloning, RT-qPCR 


\section{Introduction}

Tyrophagus putrescentiae (T. putrescentiae) can be found throughout buildings around the world such as farms, laboratories, urban environments, and food industries (Duek et al., 2001; Solarz et al., 2007), and have successfully been isolated from these places (Smrz and Jungova, 1989; Rozej et al., 2012). Recently, T. putrescentiae has been reported to be associated with host bacterial communities or symbionts in the gut, fat body or other tissues. (Hubert et al., 2012; Kopecky et al., 2014; Brown and Lloyd, 2015). The storage mites is one of the aeroallergens, can caused asthma and rhinitis in frequently sensitising individuals (Liao et al., 2013a; Fernandez-Caldas et al., 2014). In some case, Storage mites may lead to anaphylaxis caused by the ingestion of contaminated foods (Liao et al., 2013b; Yu et al., 2014).

Temperature is usually the environmental factor with the greatest effect on developmental rate of immature mites and other poikilotherms, because of their weak ability to regulate body temperature (Southwood 1978). Their body temperature is close to their habitat temperature and they are vulnerable to extreme temperature (Rinehart et al., 2005). Insects can perform normal growth and development at the appropriate temperature. If the external temperature is too high or too low, their growth and development will stagnate and even lead to their death (Trullas et al., 2007). In addition, high temperatures can cause developmental abnormalities or defects in insects (Mironidis et al., 2005).

Heat shock proteins (Hsps) are molecular chaperones which play crucial roles in protein folding, unfolding, aggregation, degradation, and transport (Tiwari et al., 2015). It is an important vector protein molecule for temperature change in insect experimental environment. Under thermal stress, the participation of Hsps in the formation of cell tolerance is conducive to improving the viability of organisms in 
adversity (Xu et al., 2011; Colinet et al., 2013). Bettencourt had treated drosophila at 30-39 ${ }^{\circ} \mathrm{C}$ and detected the expression of $\mathrm{Hsp} 70$ in different stage, found that heat resistance of flies is closely relative to the expression of $H s p 70$ (Bettencourt et al., 2007). The expression of Hsp70 gene in Bemisia tabaci female was inhibited by RNAi, and the survival number was found to be significantly lower than that in the control group (Lv et al., 2011). Those results suggested that Hsps played an important role in improving the heat resistance of insects.

To explore temperature tolerance of $T$. putrescentiae under thermal stress, we cloned the full-length of $H s p 70$ and $H s p 90$ cDNAs from $T$. putrescentiae, and measured the mRNA expression of $H s p 70$ and $H s p 90$ at different temperature using real-time quantitative polymerase chain reaction (RT-qPCR). The results will help us understand the adaptation of $T$. putrescentiae under temperature stress.

\section{Materials and methods}

\subsection{Mites}

The $T$. putrescentiae adults were collected from the storages at Nanchang suburb, Jiangxi Province, China. The mites were maintained on wheat bran (Shangdong, China) and the tailor-made plastic containers $(18 \times 11 \times 8 \mathrm{~cm})$, which was covered with a lid to prevent escape, was used to feeding mites. A $3 \mathrm{~cm}$ diameter hole was shorn on the lid for ventilation (Sánchez-Ramos et al., 2007).

We use climate-controlled incubators (RXZ-260B) to keep the rearing units for several generations at $25 \pm 0.5^{\circ} \mathrm{C}, 75 \pm 5 \%$ relative humidity (controlled by YADU ultrasonic humidifier) and dark conditions.

\subsection{Total RNA extraction and first strand cDNA synthesis}

300 female adults of $T$. putrescentiae were separated from experimental 
population. Total RNA was extracted using the TRIzol method (Invitrogen, San Diego, CA, USA) and then treated with DNase I (Tiangen, Beijing, China). Concentration and purity of the RNAs were detected by NanoDrop2000 (Thermo, USA) at $260 \mathrm{~nm}$ and $280 \mathrm{~nm}$, respectively. Finally, using 1\% agarose gel electrophoresis to check the integrity of total RNA. The first strand cDNAs were obtained using Reverse Transcription M-MLV Kit (TaKaRa, Tokyo, Japan).

\subsection{Degenerate primers and amplification of cDNA}

To amplify partial cDNA fragments of $H s p 70$ and $H s p 90$, degenerate primers (Table 1) were designed and used in PCR according to previous study (Feng et al., 2009, Yang et al., 2012). The PCR reactions use $0.1 \mu \mathrm{g}$ cDNA as templates, $0.3 \mu \mathrm{M}$ of each primer, $12.5 \mu \mathrm{L} 2 \times$ Taq polymerase Mix (Tiangen, Beijing, China) and add $\mathrm{ddH}_{2} \mathrm{O}$ to $25 \mu 1$.

The PCR programs were operated with following cycling conditions: initial denaturation step of $3 \mathrm{~min}$ at $94^{\circ} \mathrm{C}$, followed by 35 cycles of $94^{\circ} \mathrm{C}$ for $30 \mathrm{~S}, 49^{\circ} \mathrm{C}$ for $30 \mathrm{~S}$ and $72^{\circ} \mathrm{C}$ for $60 \mathrm{~s}, 72^{\circ} \mathrm{C}$ for $10 \mathrm{~min}$. The PCR products were detected with $1 \%$ agarose gel. Bands with expected size being purified by a universal DNA puritation kit, Purified DNA fragments were cloned into the pGEM-T Easy vector and transfected into Escherichia coli DH5a cells (Promega, Madison, WI, USA).The DNA inserts of the recombinant clones were confirmed by PCR with the same degenerate primers used previous and by sequencing in both directions.

Table 1. Primers used in this study.

\begin{tabular}{|c|c|c|}
\hline Experiments & Primer name & Primer sequences(5'-3') \\
\hline \multirow{3}{*}{$\begin{array}{c}\text { Full length PCR } \\
\text { amplification }\end{array}$} & TpHSP70-1F & CAAGCTACCAAACGCGACTAACC \\
\hline & TpHSP70-1R & AAAGGAGCCGCAATGTTGAATG \\
\hline & TpHSP70-2F & AAAGGAAGAGGTTGGCAAGGAA \\
\hline
\end{tabular}




\begin{tabular}{|c|c|c|}
\hline & TpHSP70-2R & CAGTTGAAGGAAGGGTAGAGTGGA \\
\hline & TpHSP90F & TACTCTACGCTTTACATCCGAACAC \\
\hline & TpHSP90R & GAACAACTTAATCAACСТCСТCСАT \\
\hline \multirow{4}{*}{ Hsp70-3'-RACE } & 3TpHSP70-1F1 & CACCAAGATGAAGGAGACCGCCGAG \\
\hline & 3TpHSP70-1F2 & CAAGAAGACCCAGGGCGAGAAGAAC \\
\hline & 3TpHSP70-2F1 & ACAAGAAGGAGGGCGAGAAGAAC \\
\hline & 3TpHSP70-2F2 & GATCGACAATGAAGCCCGTGCAG \\
\hline \multirow{4}{*}{ Hsp70-5'-RACE } & 5TpHSP70-1R1 & GATCGACTTGTTGAGCTCCTTGCC \\
\hline & 5TpHSP70-1R2 & GGCACGGGTGATCGAGGAGTAGAA \\
\hline & 5TpHSP70-2R1 & GCTGCGAATGTCCTTGCCGGTCT \\
\hline & 5TpHSP70-2R2 & CCACCGCCCAGATCGAACACCAG \\
\hline \multirow{2}{*}{ Hsp903'-RACE } & 3TpHSP90F1 & CGTCAAGAAGTGCCTGGAGCTGT \\
\hline & 3TpHSP90F2 & TGAGGAGAAGAAGAAGCGCGAGG \\
\hline \multirow{2}{*}{ Hsp90-5'-RACE } & 5TpHSP90R1 & CATGTAGACGACCTCGAAGCCAC \\
\hline & 5TpHSP90R2 & CGTCAAACAGCTCCAGGCACTTC \\
\hline \multirow{8}{*}{ Real-time PCR } & qTpHSP90F & TTCCAGTGGCGACGAGATGT \\
\hline & qTpHSP90R & TCCTCAGGCAGCTCCAGACC \\
\hline & qTpHSP70-1F & CACTAACGACAGGCGATTCTACG \\
\hline & qTpHSP70-1 & TCAACCGAACAACCACTCAAGC \\
\hline & qTpHSP70-2F & GAACTGGTCCTGCTCGATGTGAAC \\
\hline & qTpHSP70-2R & ACCCTCGAAGACTTGAATTGTGACG \\
\hline & qTpTubulinF & TATCCTCGCATTCACTTTCCTCTGG \\
\hline & qTpTubulinR & CGAAGCAGGTGTTGGTAATCTCAGC \\
\hline
\end{tabular}

\subsection{Rapid amplification of cDNA ends}

117 The rapid amplification of cDNA ends (RACE) method was applied to obtain 118 full-length cDNAs. Gene specific primers in Table 1 were designed by identified $119 H s p 70$ and Hsp90 cDNA fragments, and 5' and 3'-Full RACE Kits (TaKaRa, Tokyo, 120 Japan) were used to amplify the 5' and 3'-ends of the two genes. The nested PCR 
reactions were as following: The first-round PCR program was as follows: pre-denaturation for $180 \mathrm{~S}$ at $94^{\circ} \mathrm{C}, 35$ cycles of $94^{\circ} \mathrm{C}$ for $30 \mathrm{~S}, 60^{\circ} \mathrm{C}$ for $30 \mathrm{~S}$ and $72^{\circ} \mathrm{C}$ for $90 \mathrm{~S}$ with a final extension of $6 \mathrm{~min}$ at $72^{\circ} \mathrm{C}$. The second-round PCR program was similar to that of the first-round.

PCR products from 5'- and 3'-RACE reaction were cloned into the pGEM-T Easy vector and transfected into Escherichia coli DH5a cells (Promega, Madison, WI, USA). Six recombinant clones were identified by PCR amplification and then sequenced (Sangon, Shanghai, China).

\subsection{Confirmation of full-length cDNA sequences}

After the 5'-ends and 3'-ends sequences were obtained, the contigs were assembled to produce putative full length of HSP70 and HSP90 sequences by using the Seqman software. To verify the full length of putative HSP70 and HSP90 cDNAs, amplifying the ORF of HSP70 and HSP90 cDNAs using full length confirmation primer listed in table 1, and the PCR products cloned into pGEM-T Easy vector and sequenced. The PCR conditions and cloned methods were same with previously described.

\subsection{Bioinformatics analysis}

Blast the sequence of TpHSP70 and TpHSP9O at the National Center for Biotechnology Information (http://www.ncbi.nlm.nih.gov/BLAST/) at both nucleotide and amino acid levels by performing the BLAST program. Using Expert Protein Analysis System (http://www.expasy.org/) to analyse the amino acid sequence. Executing multiple alignments of TpHSP70 and TpHSP9O in DNAstar software (7.1 versions) to futher analys. Using ClustalX 2.0 and MEGA 5.0 constructing Neighbor-joining phylogenic tree, based on the sequences of HSP70 and HSP90 genes (Table 2 had list the sequence code). Estimate the confidence of branches produced by using 1000 replicates' Bootstrap analysis. 
Table $2 H S P 70$ and HSP90 sequences used in phylogenetic analysis

\begin{tabular}{|c|c|c|}
\hline Accession Number & Organism & Hsp70 or Hsp90 \\
\hline NP_001036837 & Bombyx mori & BmHSP70-1 \\
\hline NP_727563 & Drosophila melanogaster & DmHSP70-3 \\
\hline AIS39468 & Haemaphysalis lava & HlHsp70 \\
\hline NP_005338 & Homo sapiens & HsHSP70 \\
\hline XP_002407132 & Ixodes scapularis & IsHsp70-2 \\
\hline XP_002433656 & Ixodes scapularis & IsHsp70-1 \\
\hline ABK76338 & Marsupenaeus japonicus & MjHsp70 \\
\hline BAE39187 & Mus musculus & MmHsp70-1 \\
\hline BAE41082 & Mus musculus & MmHsp $70-2$ \\
\hline AGQ50609 & Neoseiulus cucumeri & NcHsp70 \\
\hline ADE34170 & Nilaparvata lugens & NlHsp70 \\
\hline XP_001510947 & Ornithorhynchus anatinus & OaHsp70 \\
\hline ADM83423.1 & Panonychus citri & PcHsp70-1 \\
\hline ADM83424.1 & Panonychus citri & PcHsp70-2 \\
\hline ADM83425.1 & Panonychus citri & PcHsp70-3 \\
\hline NP_077327 & Rattus norvegicus & RnHsp70 \\
\hline ACG60423 & Tetranychus cinnabarinus & TcHsp70-2 \\
\hline ACG60424 & Tetranychus cinnabarinus & TcHsp70-3 \\
\hline АВC33921 & Tetranychus urticae & TuHSP70 \\
\hline AАB97092 & Xenopus laevis & XlHsp70 \\
\hline KR479867 & Tyrophagus putrescentiae & TpHSP70-1 \\
\hline KR479868 & Tyrophagus putrescentiae & TpHSP70-2 \\
\hline AJQ31840.1 & Tyrophagus putrescentiae & TpHSP90 \\
\hline NP_001036876.1 & Bombyx mori & BmHSP90 \\
\hline ADP37710.1 & Helicoverpa armigera & HaHSP9O \\
\hline BAE44307.1 & Chilo suppressalis & CsHSP9O \\
\hline
\end{tabular}




$\begin{array}{ccc}\text { AAA96259.1 } & \text { Xenopus laeviens } & \text { XlHSP90 } \\ \text { CAA30276.1 } & \text { Drosophila melanogaster } & \text { DmHSP90 } \\ \text { AC083357.1 } & \text { Penaeus monodon } & \text { PmHSP90 } \\ \text { EEC05121.1 } & \text { Ixodes scapularis } & \text { IsHSP90 } \\ \text { XP_003745497.1 } & \text { Metaseiulus occidentalis } & \text { MoHSP90 } \\ \text { AAB23369.1 } & \text { Rattus } \text { sp. } & \text { RaHSP90 } \\ \text { AAI21063.1 } & \text { Homo sapiens } & \text { HsHSP90 } \\ \text { BAK08840.1 } & \text { Chara braunii } & \text { CbHSP90 } \\ \text { AGQ50610.1 } & \text { Neoseiulus cucumeris } & \text { NcHSP90 } \\ \text { ACF75907.1 } & \text { Tetranychus cinnabarinus } & \text { TcHSP90 } \\ \text { ADM83426.1 } & \text { Panonychus citri } & \text { PcHSP90 }\end{array}$

\section{6 mRNA expression of TpHSP70 and TpHSP90 under thermal stress}

$149 \quad$ 2.6.1 Sample collection at different stages

$150 \quad$ Separating the eggs of $T$. putrescentiae with 140 mesh sieve. These eggs were 151 continued to grow and develop, and observed every $12 \mathrm{~h}$. After proliferation for many 152 generations, about 400 eggs, 400 larvae, 200 nymphs and 200 female adults were 153 picked out for the extraction of total RNA, respectively. Each experiments group was 154 repeated for 3 times.

2.6.2 Treatments of themal stress

156 Transferred two hundred female adult mites into a $1.5 \mathrm{ml}$ centrifuge tube in brush 157 (Jiangxi, China). The tubes were sealed by 0.45 um filter membrane (BBI, China) for 158 ventilation. Then ten tubes, each with 200 mites, were treated at a target temperature 159 including $0,5,10,15,20,30,33,36,39$, and 42 for $1 \mathrm{~h}$, respectively. Each groups 160 repeat for 3 biology treatment. Mites kept at $25^{\circ} \mathrm{C}$ were served as the control. 
The qPCR assays were performed on Fx960 Real-time Quantitative PCR (BIO-RAD, USA) with alpha-tubulin gene from T. putrescentiae (GenBank accession number: AY986760) as endogenous reference. For each gene specific primer, there are three biological duplication and three technical repetition. Finally, the expression levels of TpHSP70 and TpHSP90 were calculated with the $2^{-\triangle \triangle \mathrm{Ct}}$ method (Livak and Schmittgen, 2001).

\subsection{Data analysis}

Statistical analysis was carried out using SPSS 22.0 software (Chicago, IL, USA) for ANOVA followed by Duncan's multiple comparison tests to detect statistical difference. Significance was set at a value of $P<0.05$.

\section{Results}

3.1 Sequence analysis of TpHSP70 and TpHSP9O gene

Complete cDNA sequence of TpHSP70-1 (GenBank accession number: KR479867) and TpHSP70-2 (GenBank accession number: KR479868) were 2,494 and $2354 \mathrm{bp}$ in length with ORF of 1,998 and 1,984 bp. As shown in Figure 1A and 1B, respectively. TpHSP70-1 encoded a 665 amino acid protein. The TpHSP70-1 cDNA included a 186 bp 5'untranslated region (UTR) and a 310 bp 3' UTR. The mature protein had a calculated molecular weight of $72.72 \mathrm{kDa}$ with an isoelectric point of 5.21. A possible consensus signal sequence for polyadenylation (AATAAA) was located 45 bp upstream of the poly (A) tail. TpHSP70-2 encoded a 661 amino acid protein. The TpHSP70-2 cDNA included a 168 bp 5'untranslated region (UTR) and a 173 bp 3' UTR. The mature protein had a calculated molecular weight of 72.75 $\mathrm{kDa}$ with an isoelectric point of 5.29. A possible consensus signal sequence for polyadenylation (AATAAA) was located 45 bp upstream of the poly (A) tail. The 
Hsp70-1 and Hsp70-2 were both having three typical motifs which were observed in Hsp70 proteins family.

Complete cDNA of TpHSP90 gene was deposited in GenBank (accession number: KJ820823). As shown in Figure 1C, the full length of the TpHSP90 cDNA consisted of 2,538 bp with an ORF of 2,157 bp, which encoded a 718 amino acid protein. The TpHSP90 cDNA included a $165 \mathrm{bp} 5$ 'untranslated region (UTR) located upstream of the putative start codon (ATG) and a 216 bp 3' UTR located downstream of the stop codon. The mature protein had a calculated molecular weight of $82.79 \mathrm{kDa}$ with an isoelectric point of 4.92. A possible consensus signal sequence for polyadenylation (AATTAAA) was located $15 \mathrm{bp}$ upstream of the poly (A) tail. The typical histidine kinase-like ATPases domain, which is ubiquitous in all Hsp90 family members, was located at the position of 37-181. The five typical motifs were observed in $H s p 90$ proteins family. Hsp90 was found to be highly conserved in eukaryotic organisms. The amino acid sequence of TpHSP9O has five typical motifs of Hsp90 family: NKEIFLRELISNASDALDKIR, LGTIAKSGT, IGVFGVGFYSAYLIAD, IKLYVRRVFI and GVVDSEDLPLNISRE. C-terminal "MEEVD" motif, which is specific to the cytoplasmic type of $H s p 90$ family. Comparative analysis showed that the amino acids of TpHSP9O had a high similarity of $79-81 \%$ with other species. 
A 1 createdsundayaprilpmgagaaatictcacacagcaanaattgcaangtgaacact 61 AGCTAACAaGCTAACAaGCTACCAAACGCGACTAACCGCTAACTTTTACTGCGAaAaATC

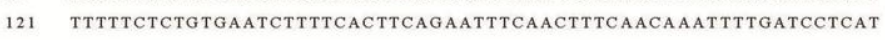
181 CAGAaAaATCACCAACCACCAACACAATGTCTCCTAAGCAGTCAAACGT TGCCATCGGCA

24 TCGACCTGTGCACGACCTACTCCTGCGTCGGTGTCTTCCAGCACGGCCAGGTGGAGATCA

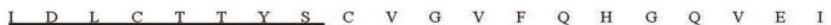

301 TCGTCAATGACCAGGGCAACCGAaCGACGCCCTCATTCGTCAGCTTCACCGACACCGAGC $\begin{array}{llllllllllllllllllllll}I & V & N & D & Q & G & N & R & T & T & P & S & F & V & S & F & T & D & T & E\end{array}$ 361 GACTGATTGGCGATGCCGCCAAGAACCAGGTGGCACTGAACCCGACGAACACCGTCTTTG $\begin{array}{llllllllllllllllllllllllll}R & \text { L } & \text { I } & G & \text { D } & \text { A } & \text { A } & \text { K } & \text { N } & \text { Q } & \text { V } & \text { A } & \text { L } & \text { N } & \text { P } & \text { T } & \text { N } & \text { T } & \text { V } & \text { F }\end{array}$ 421 ACGCGAAGCGACTGATTGGCCGCCGACTCGACGACGCCTCTGTCCAGTCCGACATGAAGC $\begin{array}{lllllllllllllllllllll}\text { D } & A & \text { K } & \text { R } & \text { L } & \text { I } & G & \text { R } & R & \text { L } & \text { D } & \text { D } & \text { A } & \text { S } & \text { V } & \text { Q } & \text { S } & \text { D } & \text { M } & \text { K }\end{array}$ 481 ACtGGTCTTTTGCAGTGGTGAaCGACAGTGGCAAGCCGAaGATCCAGGTGGAGTGCAaGG $\begin{array}{llllllllllllllllllllll}\text { H } & \text { W } & \text { S } & \text { F } & A & \text { V } & \text { V } & \text { N } & \text { D } & \text { S } & \text { G } & \text { K } & \text { P } & \text { K } & \text { I } & \text { Q } & \text { V } & \text { E } & \text { C } & K\end{array}$ 541 GCGAGACGAAACGCTTCGCCCCTGAGGAGATCTCTTCGATGGTGCTCACCAGGATGAAGG $\begin{array}{llllllllllllllllllllllll}G & E & T & K & R & F & A & P & E & E & I & S & S & M & V & L & T & R & M & K\end{array}$ 601 AGACCGCCGAGGCGTACCTGGGAACCACGGTTACCAACGCCGTCATTACCGTACCTGCTT $\begin{array}{lllllllllllllllllllllll}\text { E } & \text { T } & A & \text { E } & \text { A } & \text { Y } & \text { L } & \text { G } & \text { T } & \text { T } & \text { V } & \text { T } & \text { N } & \text { A } & \text { V } & \text { I } & \text { T } & \text { V } & \text { P } & \text { A }\end{array}$ 661 ACTTTAACGATAGCCAACGCCAGGCGACCAAGGACGCCGGAGTGATCGCCGGCCTCAACG

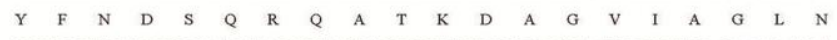
721 TGCTGCGCATCATCAACGAGCCCACTGCTGCGGCCATCGCCTACGGCCTGGACAAGAAGA $\begin{array}{llllllllllllllllllllllll}V & \text { L } & \text { R } & \text { I } & \text { I } & \text { N } & \text { E } & \text { P } & \text { T } & \text { A } & \text { A } & \text { A } & \text { I } & \text { A } & \text { Y } & \text { G } & \text { L } & \text { D } & \text { K } & \text { K }\end{array}$ 781 CCCAGGGCGAGAAGAACGTGCTCATCTTCGACCTGGGCGGCGGCACCTTCGATGTCTCCA T $\quad Q \quad G \quad$ G

841 TTCTCACCATCGAGGACGGCATCTTCGAGGTGAAGTCCACCGCCGGTGACACCCACCTCG \begin{tabular}{lllllllllllllllllllll}
\hline & L & I & E & $D$ & $G$ & I & F & E & V & K & S & T & A & $G$ & G & T & H & L
\end{tabular} 901 GTGGCGAGGACTTTGACAACCGCCTGGTGAACCACTTTGTGCAGGAGTTCAAGCGCAAGC

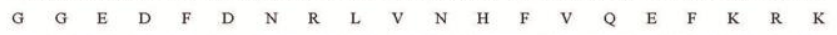
961 ACAAGAAGGACCCGTCGGACAACAAGCGCGCCCTGCGCCGTCTGCGCACCGCCTGCGAGC $\begin{array}{llllllllllllllllllllllll}\text { H } & K & K & D & P & S & D & N & K & R & A & \text { L } & R & R & L & R & T & A & C & E\end{array}$ 1021 GAGCCAAGCGAACGCTCTCCTCCTCCACCCAGGCCTCCATCGAAATCGACTCGCTCTACG $\begin{array}{llllllllllllllllllllll}R & A & K & \text { R } & \text { T } & \text { L } & \text { S } & \text { S } & \text { S } & \text { T } & \text { Q } & \text { A } & \text { S } & \text { I } & \text { E } & \text { I } & \text { D } & \text { S } & \text { L } & \text { Y }\end{array}$ 1081 AGGGCATCGACTTCTACTCCTCGATCACCCGTGCCCGCTTTGAGGAGCTCTGCGCCGACC

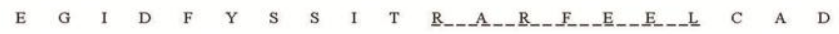
1141 TCTTCCGCTCGACGCTGGACCCGGTGGAGAATCGCTGCGCGACGCCAAGCTCGACAAGA

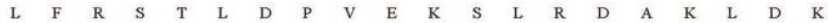
1201 ACCAGATCCACGAGATTGTCCTCGTCGGTGGCTCCACTCGCATCCCAAAGATCCAGAAGC

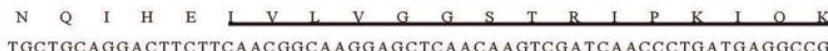
$\begin{array}{llllllllllllllllllll}\text { L } & L & Q & D & F & F & N & G & K & E & \text { L } & \text { N } & \text { K } & \text { S } & \text { I } & \text { N } & \text { P } & \text { D } & \text { E } & \text { A }\end{array}$ $\begin{array}{llllllllllllllllllll}\text { L } & \text { L } & \text { Q } & \text { D } & \text { F } & \text { F } & \text { N } & \text { G } & \text { K } & \text { E } & \text { L } & \text { N } & \text { K } & \text { S } & \text { I } & \text { N } & \text { P } & \text { D } & \text { E } & \text { A } \\ \text { TCGCCTACGGAGCCGCCGTCCAGGCTGGCCATTCTCAACGGCG ACGAGTCGGAGGCTGTCC }\end{array}$

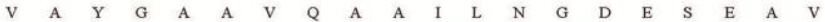
1381 AGGACCTGCTGCTGCTCGACGTGGCGCCCCTTTCGATGGGCATTGAAACTGCCGGCGGCG

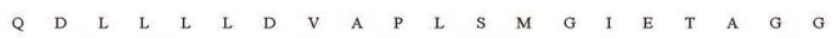
1441 TCATGACCACGCTGATCAAGCGCAACACCACCATTCCCACCAAGCAGACGCAGACCTTCT $\begin{array}{llllllllllllllllllllllll}\mathrm{V} & \mathrm{M} & \mathrm{T} & \mathrm{T} & \mathrm{L} & \mathrm{I} & \mathrm{K} & \mathrm{R} & \mathrm{N} & \mathrm{T} & \mathrm{T} & \mathrm{I} & \mathrm{P} & \mathrm{T} & \mathrm{K} & \mathrm{Q} & \mathrm{T} & \mathrm{Q} & \mathrm{T} & \mathrm{F}\end{array}$ 1501 CCACCTACTCGGACAATCAGCCCGGTGTGCTGATTCAGGTCTTTGAGGGCGAGCGAGCAA $\begin{array}{llllllllllllllllllllllll}S & T & Y & S & D & N & Q & P & G & V & \text { L } & \text { I } & Q & \text { V } & \text { F } & \text { E } & \text { G } & \text { E } & R & \text { A }\end{array}$ 1561 TGACCAAGGACAACAACCTGCTGGGAAAGTTTGAGCTGACGGGCATTCCGCCGGCACCTC $\begin{array}{llllllllllllllllllllllllll}M & \mathrm{~T} & \mathrm{~K} & \mathrm{D} & \mathrm{N} & \mathrm{N} & \mathrm{L} & \mathrm{L} & \mathrm{G} & \mathrm{K} & \mathrm{F} & \mathrm{E} & \mathrm{L} & \mathrm{T} & \mathrm{G} & \mathrm{I} & \mathrm{P} & \mathrm{P} & \mathrm{A} & \mathrm{P}\end{array}$ 1621 GCGGCGTCCCCCAGATTGAGGTCACCTTTGACATTGACGCCAACGGCATTCTCAACGTCT

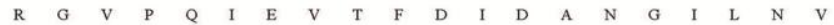
1681 CTGCCGTGGACAAGAGCACCGGCAAGCAGAACAAGATCACCATCACCAATGACAAGGGCC

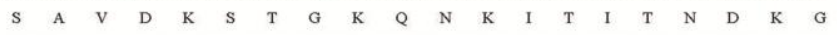
1741 GTCTGACAAAGGAGGACATTGAGCGCATGGTCAAGGAGGCCGAGTCGTACCGCGACGAGG $\begin{array}{llllllllllllllllllllllll}R & L & T & K & E & D & I & E & R & M & V & K & E & A & E & S & Y & R & D & E\end{array}$ 1801 ACGAGAAGCAGCGCGACCGCATCGCCGCCCGCAATGCCCTCGAGGGCTACGCCTTCCAGA $\begin{array}{lllllllllllllllllllll}\text { D } & \text { E } & \text { K } & \text { Q } & \text { R } & \text { D } & \text { R } & \text { I } & \text { A } & \text { A } & \text { R } & \text { N } & \text { A } & \text { L } & \text { E } & \text { G } & \text { Y } & \text { A } & \text { F } & \text { Q }\end{array}$ 1861 TCAAGTCGACGATTGAGGAGGAGGTTATCAAGTCGAAGATCTCAGAGGAGGACCGCAAGA $\begin{array}{lllllllllllllllllllll}I & K & S & T & I & E & E & E & V & I & K & S & K & I & S & E & E & D & R & K\end{array}$ 1921 AGATTAGCGAGAAGGTGGAGGAGACGGTCAAGTGGCTGGACGCGAATGCCCTCGCCGAGA

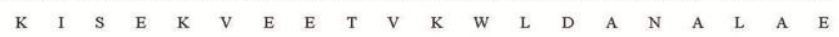
1981 AGGAGGAGTTTGAGCACCAGCGGAAGGAGCTGGAGGGCATCTGCAACCCCATCATCACCA

$\begin{array}{lllllllllllllllllllllll} & \mathrm{K} & \mathrm{E} & \mathrm{E} & \mathrm{F} & \mathrm{E} & \mathrm{H} & \mathrm{Q} & \mathrm{R} & \mathrm{K} & \mathrm{E} & \mathrm{L} & \mathrm{E} & \mathrm{G} & \mathrm{I} & \mathrm{C} & \mathrm{N} & \mathrm{P} & \mathrm{I} & \mathrm{I} & \mathrm{T}\end{array}$ 2041 AGCTGTACCAGCAGGCGGGCGGTGCCCCTGGTGGCATGCCCGGTGGAATGCCCGAAGGCT $\begin{array}{llllllllllllllllllll}\mathrm{K} & \mathrm{L} & \mathrm{Y} & \mathrm{Q} & \mathrm{Q} & \mathrm{A} & G & G & A & P & G & G & M & P & G & G & M & P & \mathrm{E} & \mathrm{G}\end{array}$ 2101 TCCCCGGCGGATTCCCCGGTGCTGCCGGCGGAGCTCCCGGTGCCGGCGGCGCCAGCTCTG $\begin{array}{llllllllllllllllllll}\text { F } & \text { P } & G & G & F & P & \text { G } & \text { A } & \text { A } & G & G & A & P & \text { G } & \text { A } & \text { G } & \text { G } & \text { A } & \text { S } & \text { S }\end{array}$ 2161 GCGACAAGTCCGGCTCTGGCCCCACCATCGAGGAGGTCGACTAAGTCTTCGCTCGCTCTC

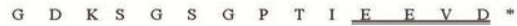

2221 GCCCTCTCTTCCCACTCTCATTCAACATTGCGGCTCCTTTAATCTTCCTGTACACACAGC 2281 ACACACCCACACCCGAGCCACCATTTTCCTCTCTCTCTCCCCTCTTTAATTCCTTCTCAC 2341 ACACACCACCACACCACACCCTCCCTTTCTGTTATTATAATTTCACTCATTCATTCTTAG 2401 CATTTCTCTTTATTCTTCTTATTCATAATACTAGCAATTATAAATTATAACACTGTATGC 2461 GAAATAAATCGGTTTGAATTTTAAAAAAAAAAAAAAAAAAAAAAAAAAAAA 
GA AA A G TCAACGTTGTCTCGTGGACAGTTCCTCTCAC 61 TCAATCTTCAGACTTTCTTCAGTTTTACTCTTCAGTGTTTTCCACTTTTCTTCTTCCTCC 121 CCTCCCCTCTCTTCTGAGGTGATTTACCTCTTTTAATAGCGGATACATTCGCCGATCTGA 181 GCCTCAGTATTATCACCTGATCGACTGAATTCCCCACCATGAAGACACTGTTCCCGGCGG $\begin{array}{lllllllllll}M & K & T & L & F & P & A\end{array}$ 241 TTCTCCTGCTGACGACGTTGCTGGTGACGATCGTCTACTAAAGGAAGAGGTTGGCAAG $\begin{array}{llllllllllllllllllllllllllll}V & \text { L } & \text { L } & \text { L } & \text { T } & \text { T } & \text { L } & \text { L } & \text { V } & \text { T } & \text { I } & \text { V } & \text { Y } & \text { S } & \text { K } & \text { E } & \text { E } & \text { V } & \text { G } & \text { K }\end{array}$ 301 AAAAGGCTGACGTCGGCACTGTGATTGGCATTGATTTAGGAACCACTTACTCTTGTGTGC

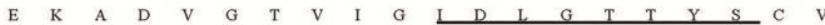
361 GCGTTTTCAAGAATGGTCGAGTGGAGATCATCGCCAACGATCAAGGTAACCGAATTACGC

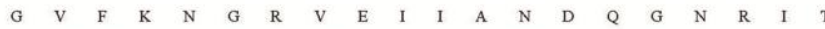
421 CCTCCTACGTGGCCTTTACCGCCGAGGGTGAGCGTCTGATTGGTGACGCGGCAAAGAACC

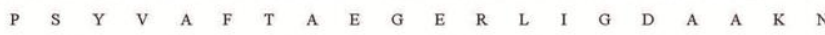
481 AGCTCACCTCGAACCCTGAGAACACCGTCTTTGACGCTAAGCGACTCATTGGACGCGAGT $\begin{array}{llllllllllllllllllllllllll}Q & \mathrm{~L} & \mathrm{~T} & \mathrm{~S} & \mathrm{~N} & \mathrm{P} & \mathrm{E} & \mathrm{N} & \mathrm{T} & \mathrm{V} & \mathrm{F} & \mathrm{D} & \mathrm{A} & \mathrm{K} & \mathrm{R} & \mathrm{L} & \mathrm{I} & \mathrm{G} & \mathrm{R} & \mathrm{E}\end{array}$ 541 GGACTGACAAAAATGTCCAACATGATCTCAAGTTCTTCCCCTTCAAAATGATTGAAAAGA

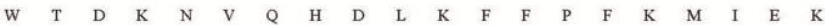
601 ACTCAAAACCACATATCAAAGTAAAGACGAGCACCGGTGATAAGGTGTTCGCCCCCGAGG $\begin{array}{lllllllllllllllllllllllll}\mathrm{N} & \mathrm{S} & \mathrm{K} & \mathrm{P} & \mathrm{H} & \mathrm{I} & \mathrm{K} & \mathrm{V} & \mathrm{K} & \mathrm{T} & \mathrm{S} & \mathrm{T} & \mathrm{G} & \mathrm{D} & \mathrm{K} & \mathrm{V} & \mathrm{F} & \mathrm{A} & \mathrm{P} & \mathrm{E}\end{array}$ 661 AGATCAGTGCCATGGTGCTGCTGAAGATGAAGGAGACCGCCGAGGCGTACCTGGGCAAGA

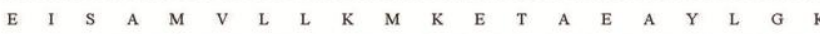
721 AGGTCACCCACGCCGTGGTCACAGTCCCTGCATATTTTAACGATGCCCAACGCCAGGCGA

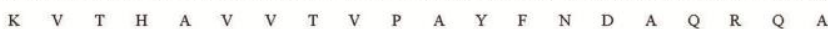
781 CGAAGGACGCCGGAGCCATTGCCGGTCTGAATGTGATGCGAATCATCAACGAGCCCACTG

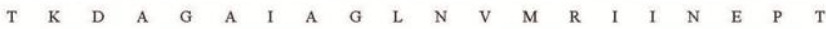
841 CCGCCGCCATTGCCTACGGTCTGGACAAGAAGGAGGGCGAGAAGAACATTCTGGTGTTCO

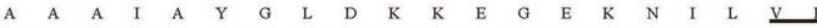
901 ATCTGGGCGGTGGCACCTTTGATGTCTCCCTGCTGACCATCGACAACGGCGTCTTTGAGG

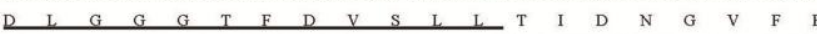
961 TGGTGGCCACCAACGGTGACAC TCACTTGGGCGGTGAGGACTTTGACCAGCGCGTGATGC $\begin{array}{llllllllllllllllllllllllllll}V & V & A & T & N & G & D & T & H & \text { L } & G & G & E & D & F & D & Q & R & V & M\end{array}$ 1021 AGCACTTTATCAAGCTGTACAAGAAAAAGACCGGCAAGGACATTCGCAGCGACAACCGAG

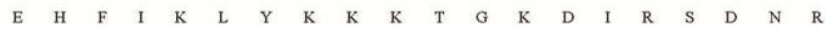
1081 CTGTGCAGAAGCTGCGTCGAGAGGTGGAGAAGGCCAAGCGAACGCTGTCCTCCGCCCACC $\begin{array}{lllllllllllllllllllllllll}A & \mathrm{~V} & \mathrm{Q} & \mathrm{K} & \mathrm{L} & \mathrm{R} & \mathrm{R} & \mathrm{E} & \mathrm{V} & \mathrm{E} & \mathrm{K} & \mathrm{A} & \mathrm{K} & \mathrm{R} & \mathrm{T} & \mathrm{L} & \mathrm{S} & \mathrm{S} & \mathrm{A} & \mathrm{H}\end{array}$ 1141 AGGCTCGCATTGAAATCGAGTCCCTCTTTGACGGCGAGGACTTCAGCGAAGTGCTCACCC

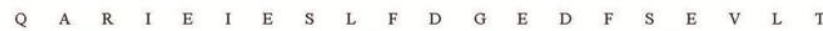
1201 GAGCCAAATTCGAGGAGCTGAACATGGACCTCTTCAGATCGACAATGAAGCCCGTGCAGA

\begin{tabular}{|lllllllllllllllllllllllll}
\hline$R$ & $A$ & $K$ & $F$ & $E$ & $E$ & $L$ & $N$ & $M$ & $D$ & $L$ & $F$ & $R$ & $S$ & $T$ & $M$ & $K$ & $P$ & $V$ & $Q$
\end{tabular} 1261 AGGTGCTCGAGGACGCGGACCTGCAGAAGAAGGACATCGATGAGATCGTCCTCGTCGGTG

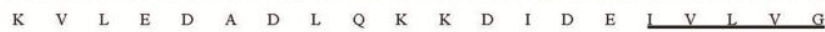
1321 GCTCCACTCGCATTCCCAAGGTGCAGCAGCTGGTGAAGGAGTTCTTCAACGGAAAGGAGC

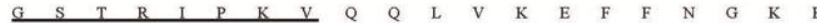
1381 CCACTCGAGGCATCAACCCTGACGAGGCCGTCGCCTTCGGTGCCGCTGTCCAGGCTGGCO

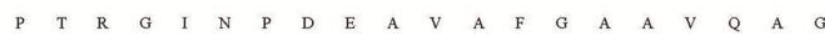
1441 TCCTCTCCGGCGAGGAGAACACCGGTGAACTGGTCCTGCTCGATGTGAACCCCCTCACCA

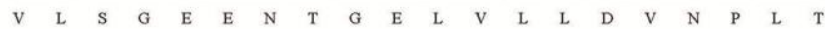
1501 TGGGCATTGAAACCGTCGGCGGTGTGATGACCAAACTGATCACCCGAAACACCGTCATCC

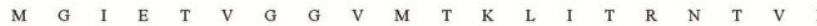
1561 CCACCAAAAAGTCGCAGATCTTCAGTACCGCTGCTGACAACCAGAACACCGTCACAATTC

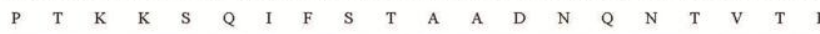
1621 AAGTCTTCGAGGGTGAACGTCCAATGACCAAAGACAACCATCAACTGGGCAAATTCGATC $\begin{array}{llllllllllllllllllllllll}Q & V & F & E & G & E & R & P & M & T & K & D & N & H & Q & L & G & K & F & D\end{array}$ 1681 TCACCGGCATCCCACCTGCCCCTCGTGGTGTTCCCCAGATTGAGGTCACCTTTGAAATT

$\begin{array}{llllllllllllllllllllllllllll}\text { L } & T & G & I & P & P & A & P & R & G & V & P & Q & I & E & V & T & F & E & I\end{array}$ 1741 ACGTCAACGGTATCCTCAAGGTTACCGCTGAGGACAAGGGCACCGGCAACAAGGAAAAGA

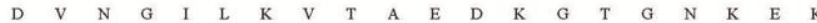
1801 TTGTCATCACCAATGACCACAATCGTCTCTCACCCGAGGACATTGAACGCATGATCAAGG $\begin{array}{llllllllllllllllllllllll} & \text { I } & V & \text { I } & \text { T } & \text { N } & \text { D } & \text { H } & \text { N } & \text { R } & \text { L } & \text { S } & \text { P } & \text { E } & \text { D } & \text { I } & \text { E } & \text { R } & \text { M } & \text { I } & \text { K }\end{array}$ 1861 ATGCGGAAAAGTTCTCCGACGAGGACAAGAAGGTGAAGGAGAAGGTTGAGGCGAAGAACC

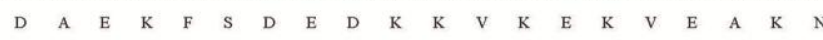
1921 AGCTCGAGTCGTATGCGTACTCACTGAAAAACCAGATCGGCGACAAGGAGAAGCTCGGTG

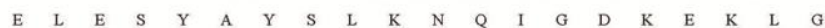
1981 GCAAACTGTCCGACGATGACAAGAAGACTATCGAGAGCTCCGTTGATGAGACCATCAAGT

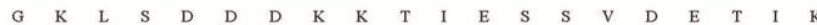
2041 GGCTCGACGCCAACGCTGATGCCGACTCTGAGGAGCTAAAGGCCCGCAAGAAGAAACTCG $\begin{array}{llllllllllllllllllllllll}\text { W } & \text { L } & \text { D } & \text { A } & \text { N } & \text { A } & \text { D } & \text { A } & \text { D } & \text { S } & \text { E } & \text { E } & \text { L } & \text { K } & \text { A } & \text { R } & \text { K } & \text { K } & \text { K } & \text { L }\end{array}$ 2101 AGGAGACCGTCACGCCCATCATCTCGAAGCTGTACGCCGGTGCCGGCGGCGACGCCGGAC

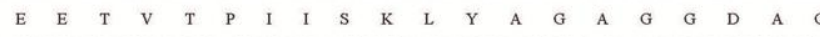
2161 AGCCGCCCGCTGAGGAGGACGCCTCGTCCAAGGACGAACTCTAGAAAAAGGTTTCCCCTT

Q P P A E E D A S S K_DEL_.

2221 TCCGTTCTCCTTTCCACTCTACCCTTCCTTCAACTGGATGTACATACGCACTACTAACAA 2281 ATTAACCAATTGGCTAGCATAGTAATC TCTTCTTTTTCTTCTCTT AATAAAAATC TCATT 2341 ATTTTACCAGATCAAAAAAAAAAAAAAAAAAAAAAAA 
GAAAACATTCGCTTGCTCTGCGTCCGGCAACGTGCTCGCTCAACGCTTTGCAACTCTCCCATTCTCCATCCCTTT GTCTCGCGTTTCGACTTGTGCGTTTAACGTTCAACGTCGTTTTACTCTACGCTTTACATCCGAACACCACCCACA CCATCATCCACGAC ATGCCTGA.AGA.ACAGCAAGAGGTCGAGACCTTCGCCTTCCAGGCCGAGATCGCCCAGTTG

ATGAGCCTGATCATCAACACTTTCTACTCGAACAAGGAGATCTTCCTGCGTGAGTTGATCTCTAACGCCTCTGAC $\begin{array}{lllllllllllllllllllllllllll}M & S & I & I & I & N & T & F & Y & S & N & R & E & I & F & I & R & E & I & I & S & N & A & S & D\end{array}$ GCCCTCGACAAAATCCGCTACGAATCTCTCACTGACCCGACCAAGATCTCCGAGGTGAAGGACTTTCACATTCGC $\begin{array}{lllllllllllllllllllllllllll}A & I & D & K & I & R & Y & E & S & I & T & D & P & T & K & I & S & E & V & K & D & F & H & I & R\end{array}$ ATCATCCCGAACAAGGATGAGA.AACGCTGACCATCATCGATACCGGCATCGGCATGACCAAGGCCGACCTGATC

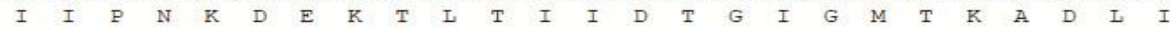
A.ACA.ATTCGGCACCATTGCCAAGTCCGGCACGAAGGCGTTCATGGAGGCCCTCCAGGCGGGCGCTGACATTTCG

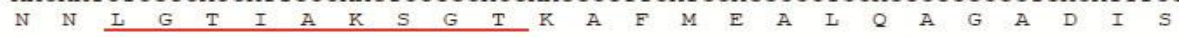
ATGATTGGCCAGTTCGGTGTGGGCTTCTACTCGGCCTACCTCATTGCCGACAAGGTGACGGTGCACTCGAAGCAC

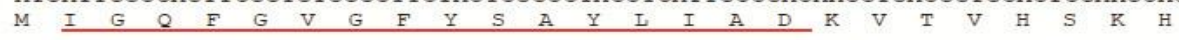
A.ATGATGACGAGCAGTACATCTGGGAGTCCTCTGCCGGCGGCTCTTTCACCATCCGCCCTGACCCCGGCGAACCG

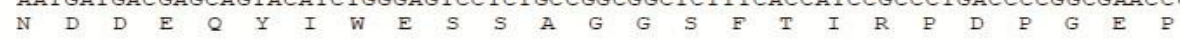
CTCGGCCGCGGCACCA.AGATTGTGCTGCACCTGAAGGAGGACCAGATCGAGTACTCCGAGGAGCGCCGCATCAAG $\begin{array}{llllllllllllllllllllllllll}I & G & R & G & T & K & I & V & I & H & I & K & E & D & Q & I & E & Y & S & E & E & R & R & I & K\end{array}$ GAGATCGTGAAGAAGCACTCGCAGTTCATTGGCTACCCCATCAAGCTGCTGGTGCAGAAGGAGCGCGAAAAGGAG

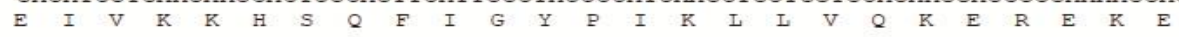

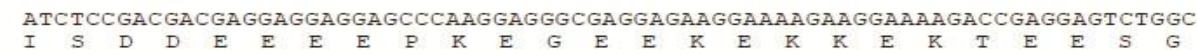
GACGAGCCCAAGGTGGAGGACGTCGAGGATGAGGCCGAGTCGAAGGAGA.A.A.AGA.A.A.AGACCATCA.AGGAGA.AG $\begin{array}{llllllllllllllllllllllllll}D & E & P & K & V & E & D & V & E & D & E & A & E & S & K & E & K & K & K & K & T & I & K & E & K\end{array}$ TACATCGAGGACGAGGAGCTGAACAAGACGAAGCCCATCTGGATGCGCA.ACCCCGATGAGATCACCCAGGACGAG TACGGCGAGTTCTACAAGTCGCTCACCAATGACTGGGATGACCACCTGGCGGTGAAGCACTTTTCGGTTGAGGGC $\begin{array}{lllllllllllllllllllllllll}Y & G & E & F & Y & K & S & L & T & N & D & W & D & D & H & I & A & V & K & H & F & S & V & E & G\end{array}$ CACTCGAGT TCCGCGCCCTGCTGTTCATTCCCAAGCGCGCCCCCTTTGATCTCTTTGAGA.ATAAGAAGCAGAA

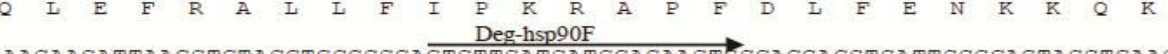

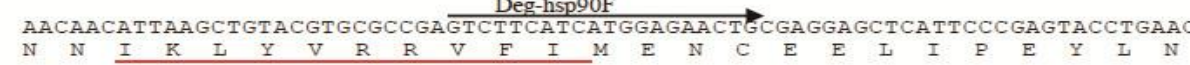
TTCGTCAAGGGCGTGGTCGACAGCGAGGACCTGCCGCTGA.ACATCTCCCGAGAGACCCTCCAGCAGAACA.AATC

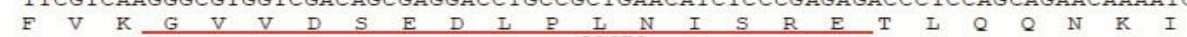
CTGAAGGTGATCCGCAAGA.ACCTCGTCAAGAAGTGCCTGGAGCTGTTTGACGA.ACTGGCCGAGGACAAGGAGAT D I A E D K E M TACAAGAAGTTCTACGAGAACTTCAGCAAGAACATGAAGCTCGGCATCCACGAGGACAGCCAGAACCGCAAGAAG

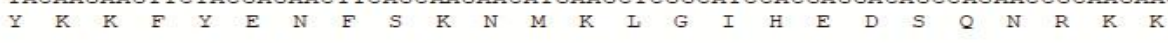
CTCGCTGAGCTGCTGCGCTACCACACCTCTTCCAGTGGCGACGAGATGTGCTCCCTGAAGGACTACGTGTCCCGC

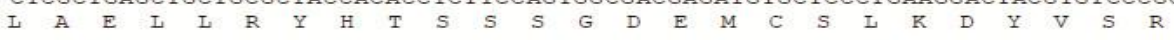
ATGA.AGGAGAACCAGA.AGGACATCTACTTCATCACCGGCGAGTCGAAGGAGATCGTTGCCAACTCGGCCTTCGTC $\begin{array}{lllllllllllllllllllllllllllll}M & K & E & N & Q & K & D & I & Y & F & I & T & G & E & S & K & E & I & V & A & N & S & A & F & V\end{array}$ GAGCGCGTCCGCAAGCGTGGCTTCGAGGTCGTCTACATGGTGGAGCCCATTGACGAGTACTGCGTCCAGCAGCTG

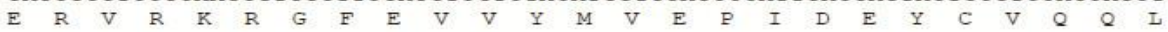

1726 AAGGAGTTCGACGGCAAGACCCTGGTCTCGGTCACCAAGGAGGGTCTGGAGCTGCCTGAGGACGAT GAGGAGA.A

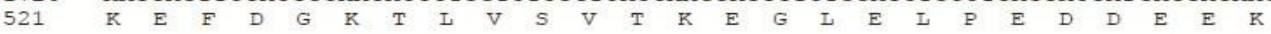
$3 G S P 2$

1801 A.GAAGCGCGAGGACGACAAGAAGA.AGTTCGAGA.ACCTGTGCAAGGTGATGAAGGACGTCCTCGAGA.AGAAGGTC $\begin{array}{llllllllllllllllllllllllll}546 & K & K & R & E & D & D & K & K & K & F & E & N & L & C & K & V & M & K & D & V & I & E & R & K & V\end{array}$

1876 GAGAAGGTGCTCGTCTCGAACCGCCTGGTCAGCTCGCCCTGCTGCATCGTCACTAGCCAGTACGGCTGGAGCGCC $571 \mathrm{E} \mathrm{K}$ V I V S N R I V S S P C C I V I S Q Y G W S A 1951 AACAT GGAGCGCATCATGAAGGCCCAGGCTCTTCGTGACTCCTCTGCCCTCGGGTACATGGCCGCGA.A.AAGAAC

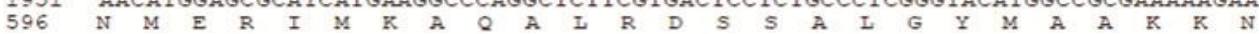
2026 CTCGAGATCAACCCGGACCACCCCATCATCGAGAACCTTCGCCTCAAGGTGGAGGCCGACAAGAACGACAAGGCC 2101 GTCAAGGACCTGGTGAACCTGCTGCTGGAGACCTCGCTGCTCTGCTCTGGTTTTGGCCTCGAGGACCCCCAGACO

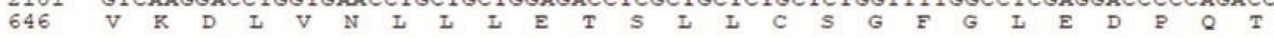
2176 CACGCCTCTCGCATCTACCGCATGATCAAGCTTGGTCTCGGCATCGATGAGGATGATCTCCCCGTCGTGGAGGAA $\begin{array}{lllllllllllllllllllllllll}H & A & S & R & I & Y & R & M & I & K & I & G & L & G & I & D & E & D & D & L & P & V & V & E & E\end{array}$ 2251 GCTCCCATTGCCGAGGAGCCCATTCCCGACGCCGAAGCTGCCGATGCTTCTCGCATGGAGGAGGTTGATTAASTI

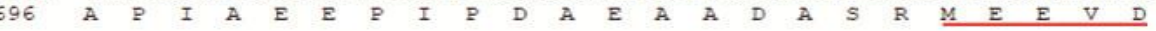

2326 GTTCTTCCACCCACCCATTAGAATTAGTATTCGCTCTCCCCTTTGTGTGTGAGATGCTTTCTCTCATTTACTGTA

2401 ATTAATATCATTTTATTTTACTTTTAACATCCACTCAACACTTGCTATTTTTCATTTGAATAGTTGATCTGGAAT 2476 CTATCTCTCACTTGAATTAAAGTGTAGAACTCTTTAAAAAAAAAAAAAAAAAAAAAAAAAAAAA 
TpHSP70-2 (B) and TpHSP90(C). Signature sequences of TpHSP genes are marked with underline.

\subsection{Homology analysis of TpHSP70 and TpHSP90}

A BLASTP (http://blast.ncbi.nlm.nih.gov/Blast.cgi) search of GenBank revealed that TpHSP70-1 and TpHSP70-2 were belong to HSP70 family. At the same time, TpHSP90 was belong to Hsp90 family. Furthermore, multiple sequence alignment showed that the deduced amino acid sequence of TpHSP70-1 and TpHSP70-2 shared high similarity with three HSP70s from Tetranychus cinnabarinus, Ixodes scapularis, Bombyx mori, Drosophila melanogaster and Homo sapiens (Figure 2A). Additionally, phylogenetic analysis showed that the two TpHSP70s from T. putrescentiae belong to two branches of phylogenetic tree respectively, in which protein TpHSP70-1 belongs to cytoplasmic type and protein $T p H S P 70-2$ belongs to endoplasmic reticulum type (Figure 3A). Meanwhile, the deduced amino acid sequence of TpHSP90 shared high similarity with five HSP90s from T. cinnabarinus, I. scapularis, B. mori, D. melanogaster and H. sapiens (Figure 2B). Among the compared Hsp90 proteins, TpHSP90 showed the highest similarity to Hsp90 from I. scapularis (81\% identity), and the lowest similarity to HSP90 from D.melanogaster ( $77 \%$ identity). HSP90 homology was high within the arthropods, especially in the signature regions of the Hsp90 family. The relationships of HSP90 displayed in the phylogenic tree were consistent with the traditional taxonomy of these species (Figure 3B). HSP90 from $T$. putrescentiae and T. cinnabarinus get together before come together with I. scapularis, M. occidentalis and N. cucumeris (Figure 3B). 
Ayrophagus_putrescentiae_HSP 70Tyrophagus_putrescentiae_HSP70-2 Panonychus_citri_HSP70 Ixodes_scapularis_HSP Bombyx_mon_HSP Tyrophagus putrescentiae_HSP70-1 Panonychus_citri_HSP70 Ixodes scapularis HSP Bombyx_mori_HSP 70 Homo_sapiens_HSP70

Tyrophagus_putrescentiae_HSP70-1 Tyrophagus_putrescentiae_HSP 70Panonychus_citri_HSP70 1xodes_scapularis_HPP Bomby_mon_HSP7

Tyrophagus_putrescentiae_HSP70Tyrophagus putrescentiae HSP 70-2 Panonychus_citri_HSP70 Ixodes_scapularis_HSP Bombyx_mori__ Homo_sapiens_HSP70

Tyrophagus_putrescentiae_HSP70-1 us_putrescentiae_HSP70-2
us citri_HSP70 Panonychus_Cifi_HSP70 Bombyx mori HSP70 Homo sapiens HSP70

Tyrophagus_putrescentiae_HSP 70Tyrophagus_putrescentiae_HSP 70-2 Panonychus_citri_HSP70 Ixodes_scapularis_HSP

Tyrophagus putrescentiae_HSP70-1 Tyrophagus_putrescentiae_HSP70-2 Panonychus_citri_HSP70 Ixodes_scapularis_HSP7 Bombyx_mori_HSP70

Tyrophagus_putrescentiae_HSP70Tyrophagus_putrescentiae_HSP 70-2 Panonychus_citri_HSP70 Ixodes_scapularis_HSP Bombx_moni_He

Tyrophagus_putrescentiae_HSP70Tyrophagu__putrescentiae_HSP 70-2 Panonychus_citri_HSP70 Ixodes_scapularis_HSP70 Bombyx_mori_HSP70 Homo_sapiens_HSP70

Tyrophagus_putrescentiae_HSP70-1 (a) Panonychus_citri_HSP70

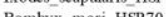
Homo sapiens_HSP70

Tyrophagus_putrescentiae_HSP70-1 Tyrophagus putrescentiae HSP70-2 Panonychus_citri_HSP70 Ixodes_scapularis_HSP70 Bombyx_mori_HSP70
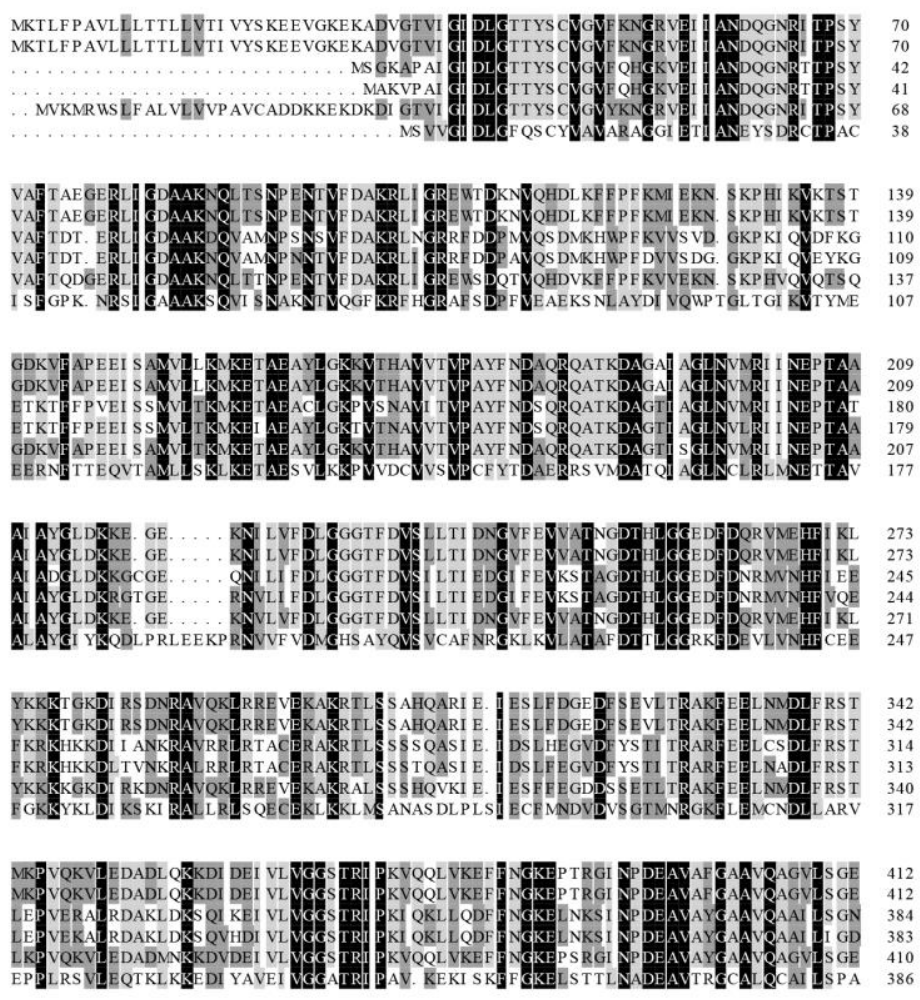
ENIGE VLLDVN KDES VQDL LLDVT PLS GI ETAGGVMTPLI MGSTTI PTKOS OI FTTYËDNOPGVII OVYEGERAMTKDN 454 KS EQVQDL LLLDVTPLS LGI ETAGGVMTVLI KRNITI PTRQTQTF TTYS DNQP GVLI QVFEGERAMT KDN 453 . QDIDAI VLLDVNPL TMGI ETVGGGMTKLI PRNTVI PT KKS QI F S TAS DNQHTVTI QVYEGERPMTKDN 478

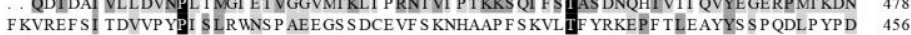

HQLGKĪDLTGI PPAPRGVPQI E. VTFEI DVNGII LKVTTAEDKGTGNKEKI VI TNDHNRLSPEDI ERMI KDA 549 HQL GKFDL TGI PPAPRGVPQI E. TTFEI DVNGI LKVT AEDKGT GNKEKI VI TNDHNRLSPEDI ERMI KDA 549 NLLGKFELSGI P PAPRGVPQI E. VTFDI NANGI LNUS AVDKS TGRENKI TI TNDKGRLS KEQI EKMVQEA 523 NLLGK ELLTGI PP APRGVPQI E. YTFDI DANG. LNVS AVDKS TGKENKI TI TNDKGRLS KEEI ERMVKDA 522 YLLGKF DL TGI P PAPRGI PQI E. YTFEI DANGI LQVS AEDKGT GNREKI VI TNDQNRLTPEDI ERMI KDA 547

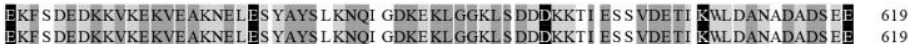
EKYKDDDE KVKDR YS AKNAL ES YCF SMKS MEE. SS AGGKI SEDDKKKI MDKVDEVI KWLDANQLAEKEE 592

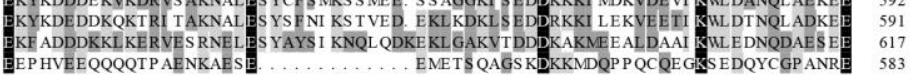

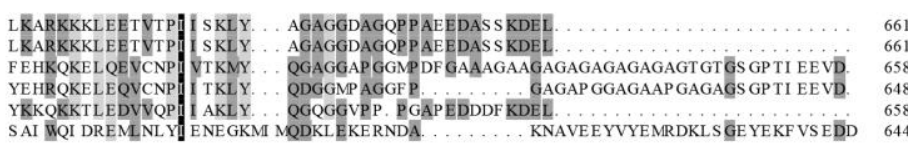
SAI WOI DREM NLY ENEGKMI MODKLEKERNDA

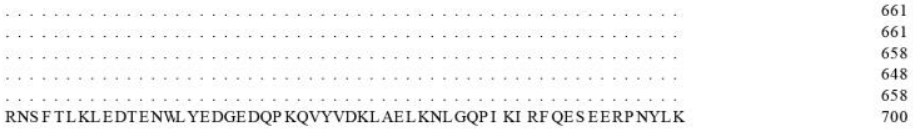




\section{B}

Tyrophagus putrescentiae HSP90 Tyrophagus_putrescentiae_
Panonychus_citri_HSP90
Ixodes scapularis_HSP90 Bombyx mori_HSP90 Homo sapiens HSP90

Tyrophagus_putrescentiae_HSP90 Panonychus_citri_HSP90 Ixodes_scapularis HSP90 Bombyx_mori_HSP99 Homo_sapiens_HSP90

Tyrophagus_putrescentiae_HSP90 Panonychus_citri_HSP90 Ixodes_scapularis_HSP90 Bombyx_mori_HSP9

Tyrophagus_putrescentiae_HSP90 Panonychus_citri_HSP90
Ixodes_scapularis_HSP90 Bombyx_mori_HSP90 Homo_sapiens_HSP90

Tyrophagus_putrescentiae_HSP90 Panonychus_citri_HSP90 Ixodes_scapularis_HSP9 Bombyx_mori_HSP90
Homo_sapiens_HSP90
MAS KL VS SRLLSLCSRYGGCSRPLUTVPQRSPTLVVGDS RRTLTQUKPTS VLF RPAGL WRPYSTPQSEPD SKDEL MNI I KNTE ATES DAETF LF QAEI AQL MTLIII NTF YS NKEI F L RELI S NS SDALDKI RYES..... L 60

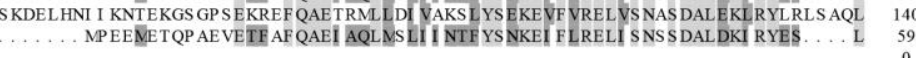

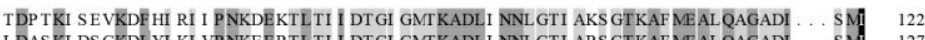

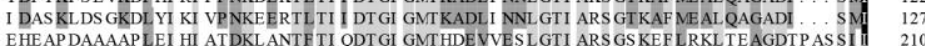

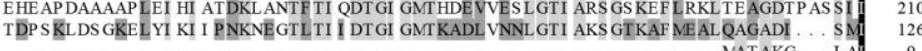

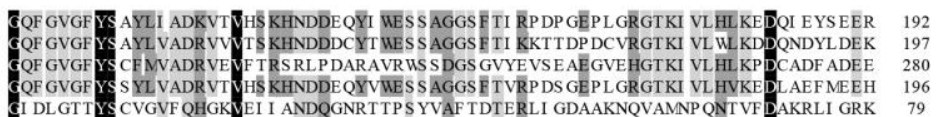

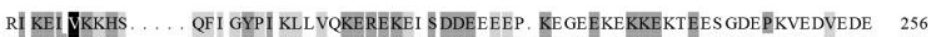
KI KEVVKKHS ..... QFI GYPI KL VVQKEREKEVS DDE GEEEKKEEDEEKKKENDEEKKDDDEEP KVEDV 26

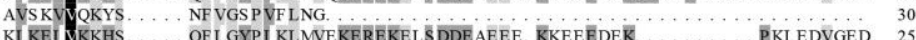
KI KEI YKKHS. ..... QFI GYPI KL MVE KEREKEL S DDEAEEE. KKKEEEDEK. ........ PKI EDVGED 250

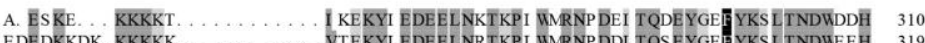

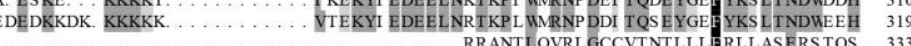

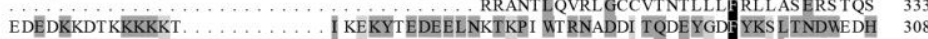
PAYF NDS QRQATKDAGVI AGL NVLRI NEPT AAAI AYGLDKGGQGERHVLI F DLGGGT DVSI LTI DDGI

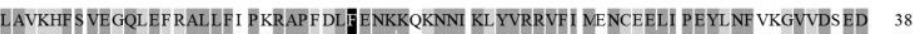
LAVKHF S VEGQLEF RALLFVPKRAPF DL E ENRKQKNNI KL YVRRVFI NDNCEDLI PEYLNFI KGVVDFED 38 GF LP HKCF AGCALF RDEKNCGWKDGF D. PQI SSAI QS SLTHKF CELFRTSLDF LHQF LASF LGI VI PSV 402 LAVKHF S VEGQLEF RAL LF VPRRRAPF DL F ENKKRKNNI KL YVRRVF I NDNCEDLI PEYL NFI RGVVDSED 378

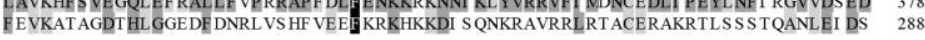

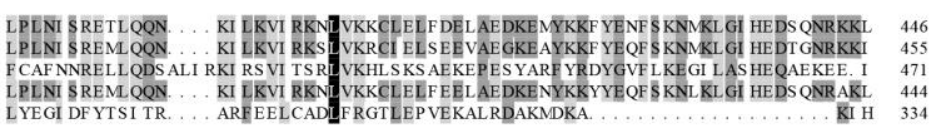

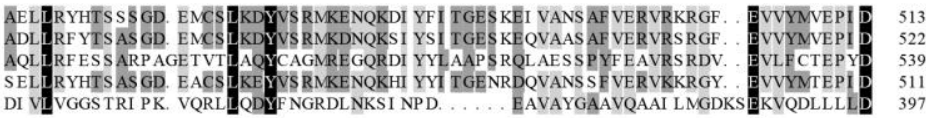

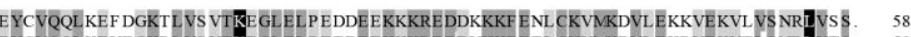

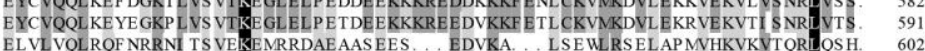

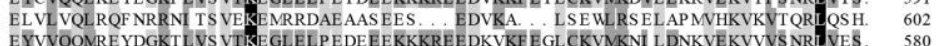

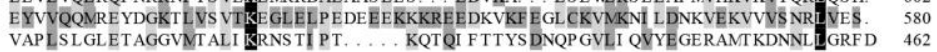

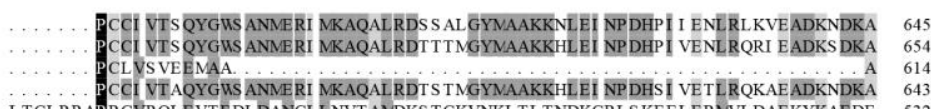

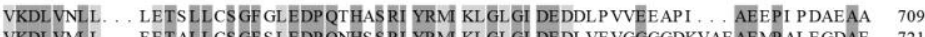
VKDL VMLL. . . FETALLCS GF SLEDPQNHS SRI YRM KLGLGI DEDL VEVGGGGDKVAE AEMPALEGDAE 721

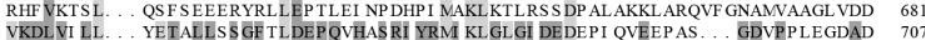
VQREKI AAKNALES YAF NMKS VVS DEGLKGKI SES DKNKI LDKCNELLS WLEVNQL. . . AEKDEF DHKRK

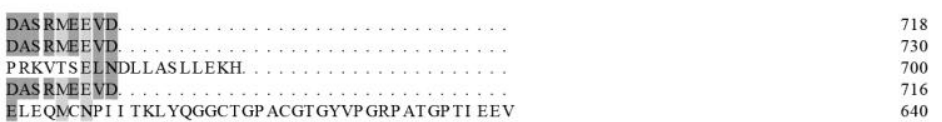

233 Figure 2 Comparison of the amino acid sequences of $H S P 70$ protein family (A) and

$234 \quad H S P 90$ protein family (B). 


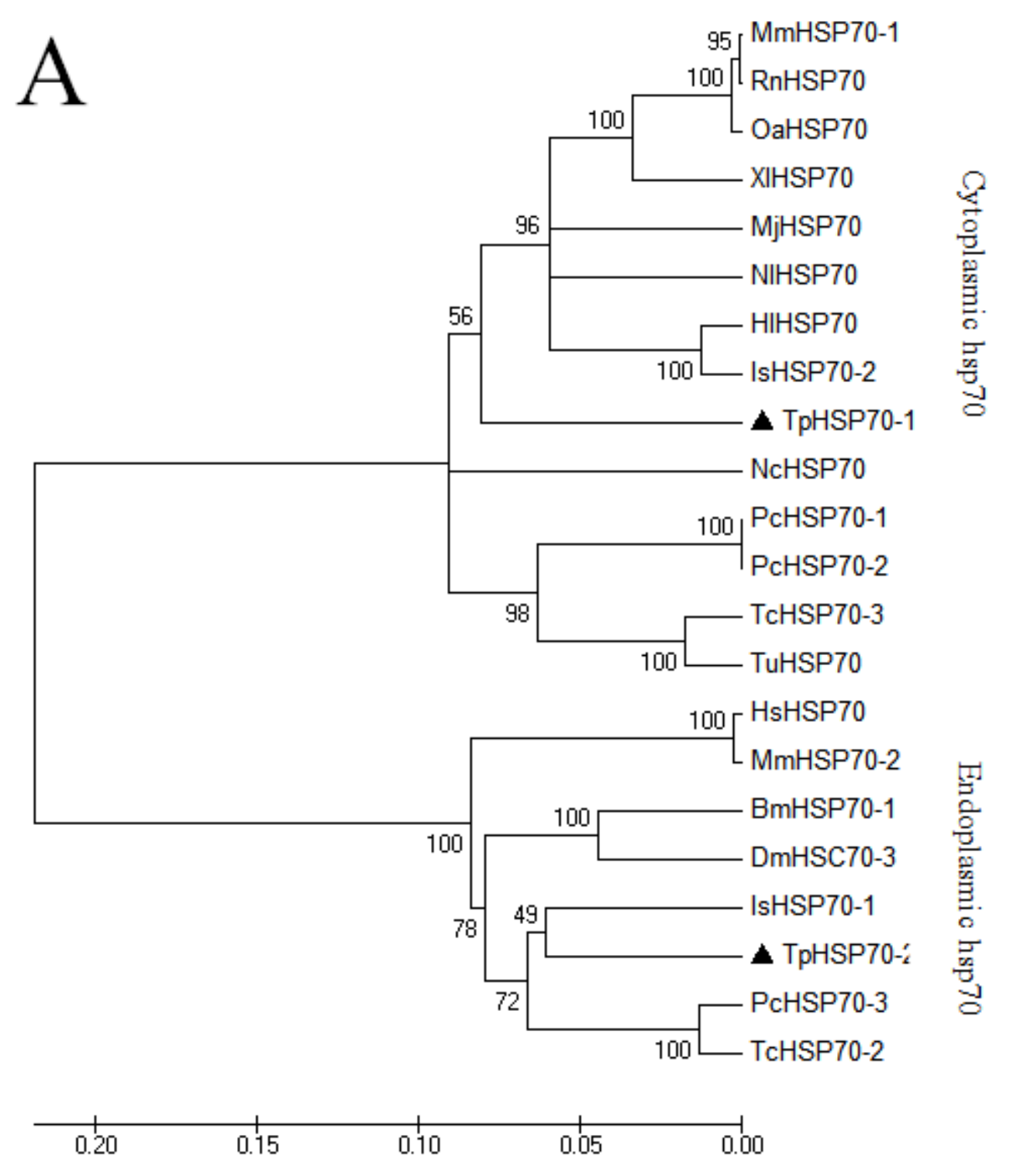




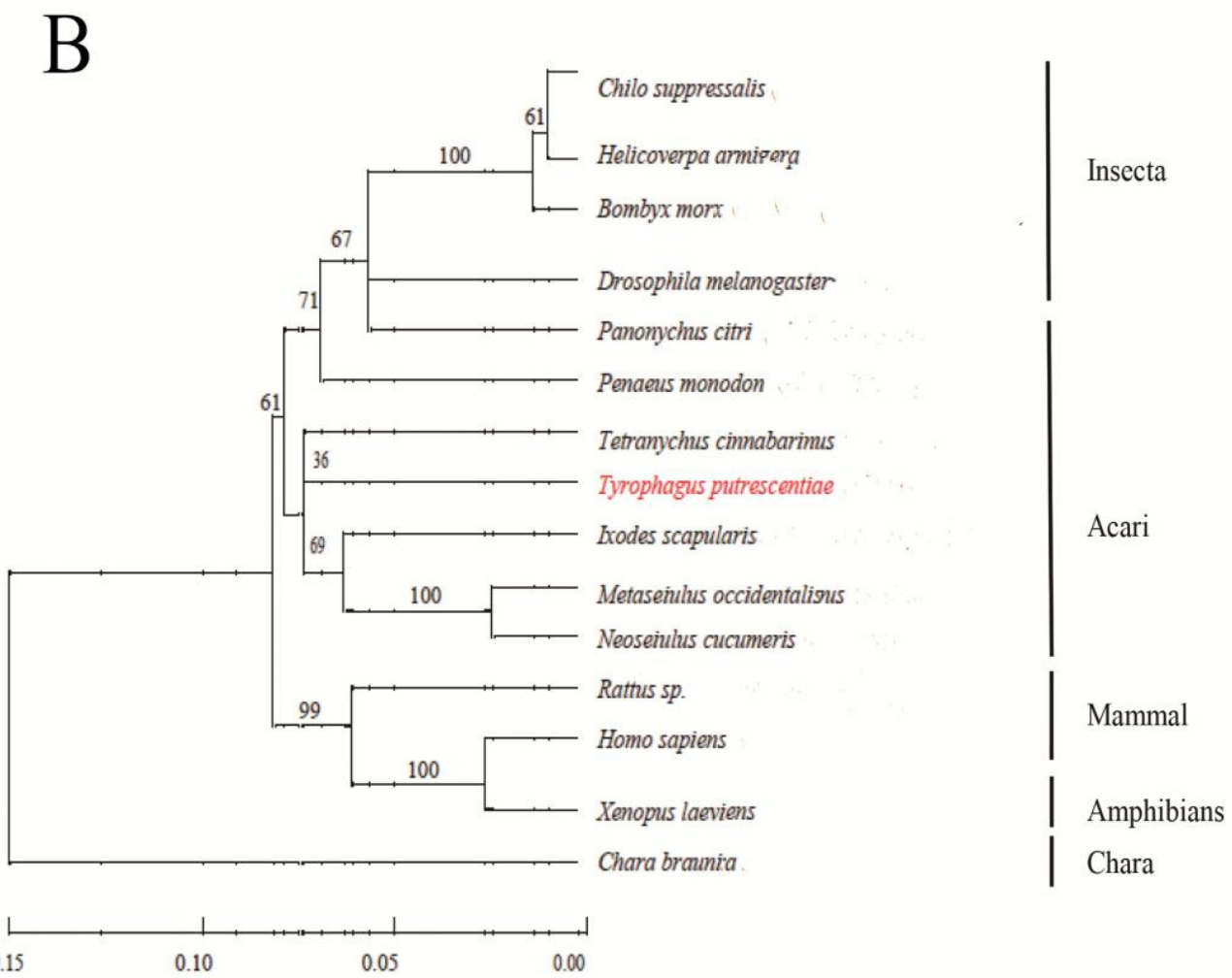

Figure 3 Phylogenetic tree of HSP70 (A) and HSP90 (B) from T. putrescentiae and other species. Constructed by the neighbor joining method based on amino acid sequences. Numbers at each branch indicate the percentage of times, and a node is supported in 1,000 bootstraps pseudo-replication by neighbor joining.

\subsection{Expression of TpHSP70 and TpHSP90 in different stages of T. putrescentiae}

Tublin was a reference gene used to measure the mRNA expression level of TpHSP90, TpHSP70-1 and TpHSP70-2 in different developmental stages of $T$. putrescentiae (egg, larva, protonymph, tritonymph and adult). As demonstrated in Figure 4, the mRNA expression level of TpHSP90, TpHSP70-1 and TpHSP70-2 246 showed rising with the development of the mites, but the expression level of three 247 HSPs in the life stages of $T$. putrescentiae were significantly different. The results 248 showed that there were significant differences in the expression levels of TpHSP70-2 249 gene $\left(\mathrm{F}_{(5,12)}=4.45, \mathrm{P}<0.05\right)$ in different developmental stages of $T$. putrescentiae. But there were no significant differences in the expression levels of TpHSP70-1 gene 
252 level of TpHSP70-1 gene in the protonymph was higher than that in other

253 developmental stages. Meanwhile, there was the lowest expression level in the egg.

254 The expression level of TpHSP70-2 genes in the tritonymph was higher than that in

255 other stages. The expression level of TpHSP90 genes in the larva was higher than that

256 in other developmental stages. Interestingly, the expression of heat shock protein gene

257 in female mites was significantly higher than that in male mites. In another word, three TpHSPs expression were decreased in the adult stage compared to the immature stages while they were still higher than those of the egg stage.

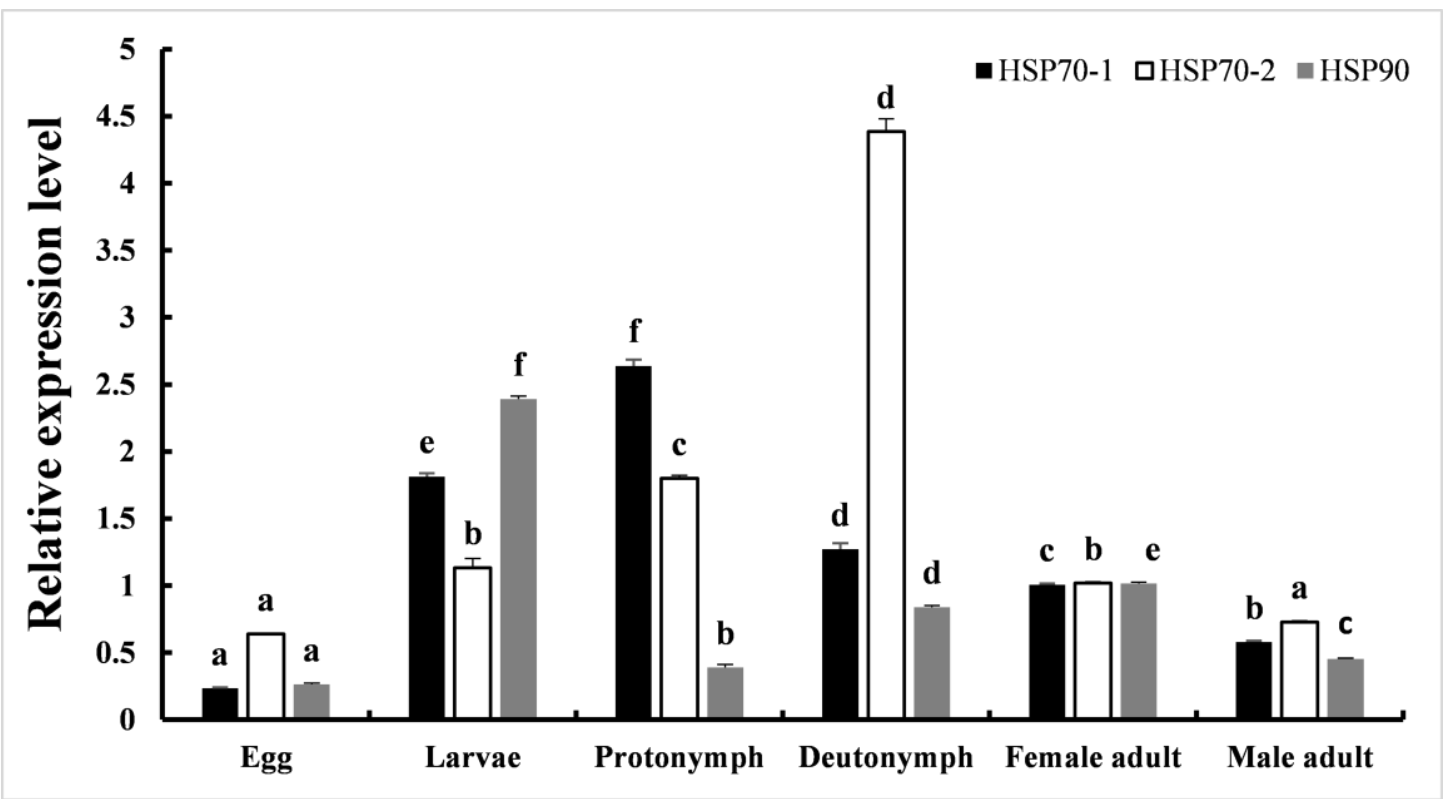

Figure 4 Stage-specific TpHSP70-1, TpHSP70-2 and TpHSP9O expression in

T.putrescentiae. The mRNA expression level of TpHSP70-1, TpHSP70-2 and

TpHSP90 in different developmental stages including egg, protonymph, deutonymph,

tritonymph and adult stages of T.putrescentiae was measured by fluorescent real-time quantitative PCR. Values are the mean $\pm S D(n=200)$. The different letters above the bars (a-f) indicate a significant difference in the means as assessed using Duncan's multiple comparison tests $(P<0.05)$. 


\subsection{Changes of TpHSP90 and TpHSP70 expression at different temperatures}

The expression pattern of TpHSP90 and TpHSP70 mRNA were examined using qPCR under different temperature conditions while the tubulin as a reference gene in low-temperature stress. Our results indicated that there were significant differences in the expression levels of three heat shock protein genes in different low-temperature stress. The expression of TpHSP70-1 gene $\left(\mathrm{F}_{(5,12)}=35.67, \mathrm{P}<0.05\right)$ increased firstly and decreased later. The expression of TpHSP70-1 gene at $10^{\circ} \mathrm{C}$ was the 6.05 times than that in the control group. The expression of TpHSP70-2 gene $\left(\mathrm{F}_{(5,12)}=179.99, \mathrm{P}<\right.$ 0.05) showed an increasing trend when the increase of temperature, and reaching the highest level at $20^{\circ} \mathrm{C}$. The expression level of TpHSP90 gene $\left(\mathrm{F}_{(5,12)}=148.64, \mathrm{P}<\right.$ 0.05 ) was the highest under $0^{\circ} \mathrm{C}$ stress. However, there was no difference between 5, 10,15 and $25^{\circ} \mathrm{C}$ (Figure $\left.5 \mathrm{~A}\right)$.

The mRNA expression level of TpHSP90, TpHSP70-1 and TpHSP70-2 in high-temperature stress were determined. The results showed that there were significant differences in the expression levels of three heat shock protein genes in different high-temperature stress (Figure 5B). The expression of TpHSP70-1 gene $\left(F_{(6,14)}=39.33, P<0.05\right) \quad$ increased firstly and decreased later. The expression level of TpHSP70-1 reached the maximum at $39^{\circ} \mathrm{C}$ and the expression was 19.73 times than the control's expression. The expression of TpHSP70-2 gene $\left(\mathrm{F}_{(6,14)}=39.27, \mathrm{P}<0.05\right)$ was the largest at $30^{\circ} \mathrm{C}$ and there was no significant difference between the control group and other temperature stresses. The expression of TpHSP90 gene $\left(\mathrm{F}_{(6,14)}=54.13\right.$, $\mathrm{P}<0.05$ ) showed its trend as similar to previous two genes. The expression level of TpHSP90 gene was 4.17 times at $42^{\circ} \mathrm{C}$ than that of the control group. 


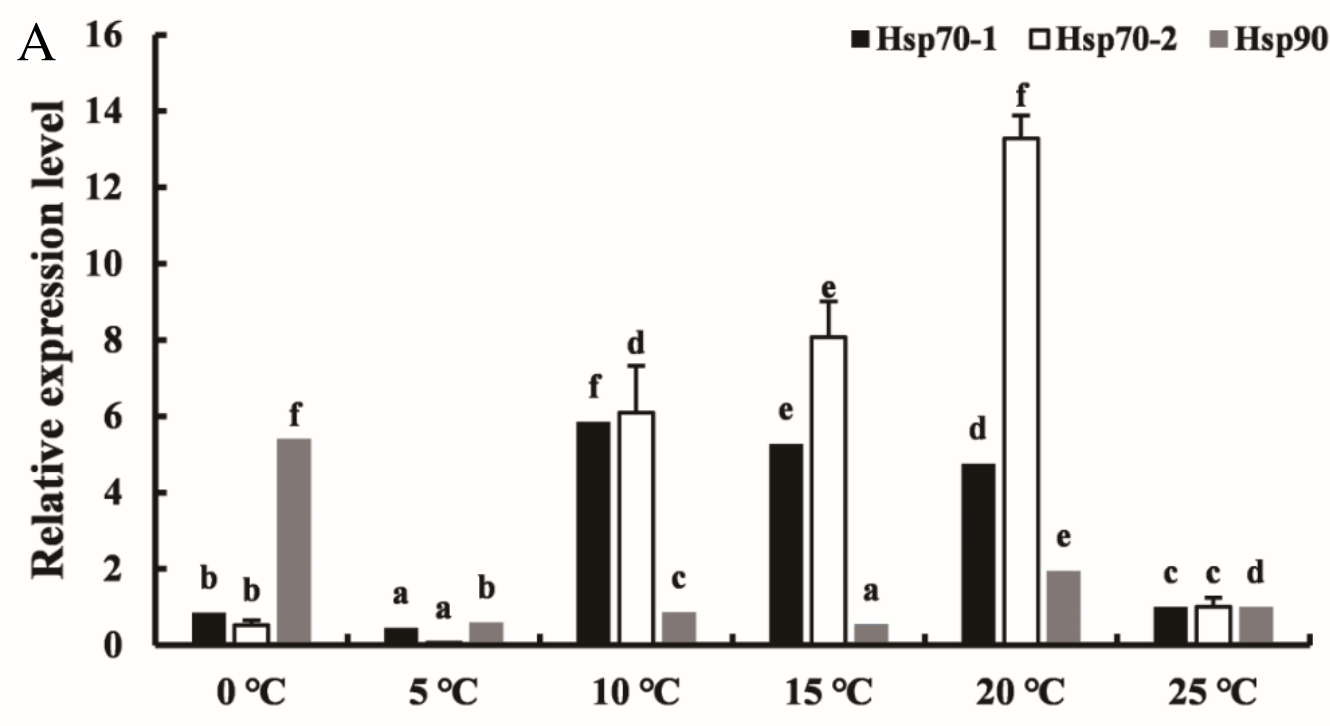

Figure 5 (A) Comparative quantitative RT-PCR analyzed of the relative expression of TpHSP 70-1, TpHSP 70-2 and TpHSP 90 at low temperatures. Control: $25^{\circ} \mathrm{C}$; Low heat shock temperature: $0^{\circ} \mathrm{C}, 5^{\circ} \mathrm{C}, 10^{\circ} \mathrm{C}, 15^{\circ} \mathrm{C}, 20^{\circ} \mathrm{C}$.

(B) Comparative quantitative RT-PCR analyzed of the relative expression of TpHSP 70-1, TpHSP 70-2 and TpHSP

29890 at high temperatures. Control: $25^{\circ} \mathrm{C}$; High heat shock temperature: $30^{\circ} \mathrm{C}, 33^{\circ} \mathrm{C}$, $36^{\circ} \mathrm{C}, 39^{\circ} \mathrm{C}, 42^{\circ} \mathrm{C}$. Each temperature treatment was three replicates. Data were represented as the mean \pm SD $(n=200)$. Letters above columns indicate levels of difference significance at $P<0.05$. The same letters are not significantly different, 
$P>0.05$.

\section{Discussion}

T. putrescentiae, a storage mite, which are colonises different human-related habitats and feeds on various post-harvest foods, was a major cause of allergic diseases (Liao et al., 2013a, 2013b; Yu et al., 2014). Administration with a crude extract of $T$. putrescentiae induced airway allergic inflammation in BALB/c mice (Liao et al., 2013a). One recent experimental study has shown that intranasal exposure to T. Putrescentiae induces an allergic inflammation in the mice. (Nuñez et al., 2016). All these call for urgent research into the prevention of the mite. In the present study, we cloned the three full-length cDNAs of HSP70-1, HSP70-2 and HSP90 genes and evaluated their expression in response to thermal stress, hoping to understand how they withstand extreme environmental temperature.

Temperature is one of the important environmental factors. A large number of studies have found that heat stress can induce the expression of HSPs genes in $D$. melanogaster (Colinet et al., 2013), S. exigua (Xu et al., 2011), T. cinnabarinus (Feng et al., 2010; Li et al., 2009; Feng et al., 2009) and P. citri (Tian et al., 2015; Yang et al., 2012). The heat resistance of organisms is closely related to heat shock protein synthesis. The result shows that the synthesized HSPs in organisms can help them to obtain the adaptability of high temperature environment, thus improving the thermal tolerance (Zhang et al., 2008). The results showed that rapid cold training increased the survival rate of Locusta migratoria. (Wang et al., 2003).

Tubulin was selected as an internal reference to detect the mRNA expression of TpHSP70-1, TpHSP70-2 and TpHSP90 gene of female adult mite of T. putrescentiae under heat stress. At the same time, the expression of three genes at different development stages in T. putrescentiae were also detected. In this study, it was found 
that under cold excitation, TpHSP70-1 and TpHSP70-2 failed to express, which is similar to Hsp70 expression of T. cinnabarinus (Li et al., 2009; Feng et al., 2009), P. citri (Yang et al., 2012) and D. melanogasteret (Colinet et al., 2013) .Our results suggested that there may be other mechanisms in $T$. putrescentiae to resist low temperature stress. Such as synthesis of trehalose, polyols and other small molecules, antioxidant reaction and production of other heat shock proteins (Liu et al., 2014; Yang et al., 2011)

The expression of T. putrescentiae TpHSP70-1 and TpHSP90 gene was up-regulated with the increase of temperature, reaching significant level. In particular, the relative mRNA expression level of TpHSP70-1 gene at $39^{\circ} \mathrm{C}$ was 19.03 times than that of the control group. This result indicates that TpHSP7O- 1 and TpHSP9O plays a certain role in improving the heat resistance of T. putrescentiae. Studies have shown that the death time of Bactrocera umbrosa's eggs and Pandemis heparana's larvae at $46^{\circ} \mathrm{C}$ is significantly higher than the control (Zhang et al, 2008; Beckett 1997). Jang et al. suggested that exposure of the Ceratitis capitata to sublethal temperatures of $42^{\circ} \mathrm{C}$ for $1 \mathrm{~h}$ enhanced heat resistance (Jang 1992). Anastrepha suspensa raised at $30^{\circ} \mathrm{C}$ had higher heat resistance than those raised at $20^{\circ} \mathrm{C}$ (Hallman 1992). After a short period of high temperature stress, the survival time of Cydia pomonella at lethal temperature was prolonged (Wang et al., 20012).

Studies have shown that the synthesis of heat shock protein began to decline after reaching a certain threshold (Didomenico et al., 1982). In this study, it was found that the expression level of TpHSP70-1 was the highest at $39^{\circ} \mathrm{C}$ and decreased at $42^{\circ} \mathrm{C}$, which may be due to its proximity to the threshold range of stress (Liu et al., 2014; Yang et al., 2011). The result indicates that heat shock protein can protect biological cells within a certain limit (Liu et al., 2014). On the one hand, induced high 
expression of heat shock protein can improve the heat resistance of insects and prevent direct damage to insects under high temperature stress. On the other hand, synthesis of heat shock protein affects the synthesis of other proteins in the insect body, forcing insects to pay corresponding costs such as shortened life span and reduced fertility (Huang et al., 2007; Chen et al., 2014). Our research shows that it was also found that the number of eggs laid by $T$. putrescentiae decreased significantly after being treated at temperatures above $39^{\circ} \mathrm{C}$.

In addition to responding to heat stress, heat shock protein genes are also involved in normal physiological activities and fertility, such as folding new peptide chains to form mature proteins in time, formation of gametes, and cell differentiation (Huot et al., 1996; Vrijenhoek 1994). Our study showed that the transcription level of The HSP90 gene of T. putrescentiae was significantly different in each developmental stages, and its expression level varied from the different developmental stages. At the same time, the expression level was the highest in the larvae's stage. This result suggests that this gene may be involved in the regulation of the growth and development of $T$. putrescentiae. The expression of $H s p 70$ gene in $T$. putrescentiae fluctuates with the development period, indicating that $H s p 70$ in T. putrescentiae also involved in normal physiological activities and reproductive development. Interestingly, the mRNA expression level of heat shock protein genes of female adult T. putrescentiae was higher than that of male. Our results were similar to the Grapholita molesta, reflecting the different ability of $T$. putrescentiae of different genders to cope with environmental temperature stress (Chen et al., 2014).

In conclusion, the new TpHSP90, TpHSP70-1and TpHSP70-2 genes sequences were isolated from T. putrescentiae, and their phylogeny with other mites were inferred. These three TpHSPs gene were differentially expressed in the developmental 
stages of T. putrescentiae, with a decreased expression in the adult stage. Our results also confirmed that these three TpHSPs are the important genes for T. putrescentiae to defend against temperature. Thus, this study will help to better understand the resistant fitness of mites and other insects under environmental stress, and guide $T$. putrescentiae management using different temperatures in crops.

\section{Acknowledgments}

This work was supported by National Natural Science Foundation of China (31760621, 31460553), Natural Science Foundation of Jiangxi Province, China (20161ACB20003, 20181BAB20405), Leadership in Major Subjects Project of Jiangxi Province (20172BCB22004), Jiangxi Key Research \& Development Plan, China(20161BBF60117), Jiangxi provincial department of education technology plan (KJLD14014), Jiangxi provincial department of education science and technology research project (GJJ14167). The authors alone are responsible for the content and writing of the paper.

\section{Disclosure statement}

No potential conflict of interest was reported by the authors.

\section{Funding}

This work was supported by National Natural Science Foundation of China (31760621, 31460553), Natural Science Foundation of Jiangxi Province, China (20161ACB20003, 20181BAB20405), Leadership in Major Subjects Project of Jiangxi Province (20172BCB22004), Jiangxi Key Research \& Development Plan, China(20161BBF60117), Jiangxi provincial department of education technology plan (KJLD14014), Jiangxi provincial department of education science and technology research project (GJJ14167). 


\section{References}

Abbar, S., Schilling, M.W., Phillips, T.W., 2016. Time-Mortality Relationships to Control Tyrophagus putrescentiae (Sarcoptiformes: Acaridae) Exposed to High and Low Temperatures. J. Econ. Entomol. 5, 2215-2220. https://doi.org/10.1093/jee/tow159

Bettencourt, B.R., Hogan, C.C., Nimali, M., 2007. Polyglutamine expansion in Drosophila: thermal stress and $H s p 70$ as selective agents. Journal of biosciences, 32(3), 537-547. https://doi.org/ 10.1007/s12038-007-0053-9

Brown, A.N., Lloyd, V.K., 2015 Evidence for horizontal transfer of Wolbachia by a Drosophila mite. Exp. Appl. Acarol. 66, 301-311. https://doi.org/10.1007/s10493-015-9918-z

Caplan. A.J., 1999. Hsp90's secrets unfold: new insights from structural and functional studies. Trends. Cell. Biol. 9, 262-268. https://doi.org/10.1016/s0962-8924(99)01580-9

Chen. B., Zhong. D., Monteiro. A., 2006. Comparative genomics and evolution of the HSP90 family of genes across all kingdoms of organisms. BMC. Genomics. 7,156. https://doi.org/10.1186/1471-2164-7-156

Colinet. H., Siaussat. D., Bozzolan. F., Bowler. K., 2013. Rapid decline of cold tolerance at young age is associated with expression of stress genes in Drosophila melanogaster. Journal of Experimental. Biology. 216(2), 253-259. https://doi.org/110.1242/jeb.076216

Csermely. P., Kajtár. J., Hollósi. M., Oikarinen. J., Somogyi. J., 1994. The 90 kDa heat shock protein (Hsp90) induces the condensation of the chromatin structure. Biochemical. Biophys. Research. Community. 3, 1657-1663. https://doi.org/10.1006/bbrc.1994.2124 
Duek. L., Kaufman. G., Palevsky. E., Berdicevsky. I., 2000. Mites in fungal cultures. Mycoses. 44,390-394. https://doi.org/10.1046/j.1439-0507.2001.00684.x

Feng. H., Wang. L., Liu. Y., He. L., Li. M., Lu. W., Xue. C., 2010. Molecular charac-terisation and expression of a heat shock protein gene (HSP90) from the carmine spider mite, Tetranychus cinnabarinus (Boisduval). Journal of Insect. Science. 10,112. https://doi.org/10.1673/031.010.11201

Feng. Y., Dearen. T., Cama. V., Xiao. L., 2009. 90-kilodalton heat shock protein, Hsp90, as a target for genotyping Cryptosporidium spp. known to infect humans. Eukaryot. Cell. 8(4),478-482. https://doi.org/10.1128/EC.00294-08

Fernandez-Caldas. E., Puerta. L., Caraballo. L., 2014. Mites and allergy. Chem Immunol Allergy. 100,234-242. https://doi.org/10.1159/000358860

Gupta. R.S., 1995. Phylogenetic analysis of the $90 \mathrm{kD}$ heat shock family of protein sequences and an examination of the relationship among animals, plants, and fungi $\quad$ species. Mol. Biol. Evol. 6,1063-1073. https://doi.org/10.1093/oxfordjournals.molbev.a040281

Han., Ying. Li., Hou., Cong-Cong., Du., Chen., Zhu., Jun-Quan..,Molecular cloning and expression analysis of five heat shock protein 70 (HSP70) family members in Lateolabrax maculatus with Vibrio harveyi infection. Fish \& Shellfish Immunology. 60, 299-310. https://doi.org/10.1016/j.fsi.2016.11.056

Hubert. J., Kopecky. J., Perotti. M.A., Nesvorna. M., Braig. H.R., Sagova-Mareckova. M., 2012. Detection and identification of species-specific bacteria associateed with synanthropic mites. Microb. Ecol.. 63,919-928. https://doi.org/10.1007/s00248-011-9969-6

Jiang. X.F., Zhai. H.F., Wang. L., Luo. L.Z., Sappington. T.W., Zhang. L., 2012. Cloning of the heat shock protein 90 and 70 genes from the beet armyworm, 
Spodoptera exigua, and expression characteristics in relation to thermal stress and development. Cell. Stress. Chaperones. 17, 67-80. https://doi.org/10.1007/s12192-011-0286-2

Kopecky. J., Nesvorna. M., Mareckova-Sagova. M., Hubert. J., 2014. The effect of antibiotics on associated bacterial community of stored product mites. PLOS. ONE. 9,e112919. https://doi.org/10.1371/journal.pone.0112919.

Liao. E.C., Ho. C.M., Yin. S.C., Tsai. J.J., 2013a. Immune responses to Tyrophagus putrescentiae induced airway inflammation in mice. J. Investig. Allergol. Clin. Immunol. 23, 20-29. https://doi.org/10.1159/000350402.

Liao. E.C., Lin. Y.H., Chiu. C.L., Lin. T.C., Tsai. J.J., 2013b. Identification of allergenic component Tyr $\mathrm{p} 8$ from Tyrophagus putrescentiae and cross-reactivity with Der p 8. Clin. Vaccine. Immunol. 20,506-512. https://doi.org/10.1128/cvi.00633-12

Li-Hong. Yang., Hong-Bo. Jiang., Yong-Hua. Liu., Wei. Dou., Jin-Jun. Wang., 2012. Molecular characterization of three heat shock protein 70 genes and their expression profiles under thermal stress in the citrus red mite. Mol. Biol. Rep. (2012) 39,3585-3596. doi:10.1007/s11033-011-1132-4

Livak. K.J., Schmittgen. T.D., 2001. Analysis of relative gene expression data using real-time quantitative PCR and the 2(-Delta Delta C (T)) Method. Methods. 4,402-408. https://doi.org/10.1006/meth.2001.1262

Lü. Z.C., Wan. F.H., 2011. Using double-stranded RNA to explore the role of heat shock protein genes in heat tolerance in Bemisia tabaci (Gennadius). Journal of Experimental Biology. 214(5), 764-769. 10.1242/jeb.047415

Mironidis. G.K., Savopoulou. S.M., 2010. Effects of heat shock on survival and reproduction of Helicoverpa armigera (Lepidoptera: Noctuidae) adults. Journal 
of Thermal. Biology. 35(2), 59-69. https://doi.org/10.1016/j.jtherbio.2009.11.001

478

479

480

481

482

483

484

485

486

487

488

489

490

491

492

493

494

495

496

497

498

499

500

501

Nuñez. N.K., da Cunha. A.A., Dos. Santos. Dutra. M., Barbosa. G.L., Morassutti. A.L., de Souza R.G., Vargas. M.H., Antunes. G.L., Silveira. J.S., da Silva. G.L., Pitrez. P.M., 2016. Acute and chronic exposure to Tyrophagus putrescentiae induces allergic pulmonary response in a murine model. Asia. Pac. Allergy. 1,48-55. https://doi.org/10.5415/apallergy.2016.6.1.48

Pearl. L.H., Prodromou. C., Workman. P., 2008. The Hsp90 molecular chaperone: an open and shut case for treatment. Biochem. J. 410, 439-453. https://doi.org/10.1042/bj20071640

Peng. G., Zhao. W., Shi. Z., Chen. H., Liu. Y., Wei. J., Gao. F., 2016. Cloning HSP70 and HSP9O genes of kaluga (Huso dauricus) and the effects of temperature and salinity stress on their gene expression. Cell. Stress. Chaperones. 2, 349-359. https://doi.org/10.1007/s12192-015-0665-1

Piiroinen. S., Lyytinen. A., Lindström. L., 2013. Stress for invasion success Temperature stress of preceding generations modifies the response to insectic-ide stress in an invasive pest insect. Evol. Appl. 2, 313-323. https://doi.org/10.1111/eva.12001

Rinehart. J.P., Yocum. G.D., Denlinger. D.L., 2000. Thermotolerance and rapid cold hardening ameliorate the negative effects of brief exposures to high or low temperatures on fecundity in the flesh fly, Sarcophaga crassipalpis. Physiological. Entomology. 25(4), 330-336. https://doi.org/10.1111/j.1365-3032. 2000.00201.x

Roy. N., Nageshan. R.K., Ranade. S., Tatu. U., 2012. Heat shock protein 90 from neglected protozoan parasites. Biochim. Biophys. Acta. 3,707-711. https://doi.org/10.1016/j.bbamcr.2011.12.003

Rozej. E., Witalinski. W., Szentgyorgyi. H., Wantuch. M., Moron. D., Woyciechowski. 
M., 2012. Mite species inhabiting commercial bumblebee (Bombus terrestris) nests in Polish greenhouses. Exp. Appl. Acarol. 56, 271-282. https://doi.org/10.1007/s10493-012-9510-8

Sánchez-Ramos. I., Alvarez-Alfageme. F., Castañera. P., 2007. Effects of relative humidity on development, fecundity and survival of three storage mites. Exp. Appl. Acarol. 1-2, 87-100. https://doi.org/10.1007/s10493-007-9052-7

Smrz. J., Jungova. E., 1989. The ecology of a field population of Tyrophagus putrescentiae (Acari. Acaridida). Pedobiologia. 33, 183-192. https://doi.org/10.2307/3565906

Solarz. K., Senczuk. L., Maniurka. H., Cichecka. E., Peszke. M., 2007. Comparisons of the allergenic mite prevalence in dwellings and certain outdoor environments of the Upper Silesia (southwest Poland). Int. J. Hyg. Environ. Health. 210, 715-724. https://doi.org/10.1016/j.ijheh.2006.11.007

Southwood. TRE., 1979. Ecological methods, with particular reference to the study of insect populations. Chapman and Hall, London. Ecology. 60(6), 1290. https://doi.org/10.2307/1936978

Sun. Y., Sheng. Y., Bai. L., Zhang. Y., Xiao. Y., Xiao. L., Tan. Y., Shen. Y., 2014. Ch-aracterizing heat shock protein 90 gene of Apolygus lucorum (Meyer-Dür) and its expression in response to different temperature and pesticide stresses. Cell. Stress. Chaperones. 5, 725-739. https://doi.org/10.1007/s12192-014-0500-0

Thomas. W.B., 1996. Methyl bromide: effective pest management tool and environmental threat. J. Nematol. 28(4S), 586-589. https://doi.org/10.2307/1565709

Tianrong. Xin., Tao. Lian., Xiaoyue. Li., Lei. Li., Shengquan. Que., Xinyu. Liu. \& Bin. Xia., 2018. Gene cloning and expression of heat shock protein gene from 
Aleuroglyphus ovatus and its response to temperature stress. International Journal of Acarology. 44(7), 279-287.

https://doi.org/ $10.1080 / 01647954.2018 .1446053$

Tiwari. S., Thakur. R., Shankar. J., 2015. Role of Heat-Shock Proteins in Cellular Function and in the Biology of Fungi. Biotechnol. Res. Int. 11. https://doi.org/10.1155/2015/132635

Trullas. S.C., van. Wyk. J.H., Spotila. J.R., 2007. Thermal melanism in ectotherms. Journal of Thermal. Biology. 32(5), 235-245. https://doi.org/10.1016/j.jtherbio.2007.01.013

Wei-Wei. Liu., Pu. Yang., Xiao-Ming. Chen., Dong-Li. Xu., and Yan-Hong. Hu.,2014. Cloning and Expression Analysis of Four Heat Shock Protein Genes in Ericerus pela (Homoptera: Coccidae).Journal of Insect Science. 14(142), 2014; DOI: 10.1093/jisesa/ieu032

Whitesell. L., Lindquist. S.L., 2005. HSP90 and the chaperoning of cancer. Nat. Rev. Cancer. 5, 761-772. https://doi.org/10.1038/nrc1716

Xu. Q., Zou. Q., Zheng. H., Zhang. F., Tang. B., Wang. S., 2011. Three heat shock proteins from Spodoptera exigua: gene cloning, characterization and comparative stress response during heat and cold shocks. Comparative Biochemistry and Physiology Part B: Biochemistry and Molecular Biology. 159(2), 92-102. https://doi.org/10.1016/j.cbpb.2011.02.005

Ying. Shen., Jun. Gu. „Li-Hua Huang., Si-Chun. Zheng., Lin. Liu., Wei-Hua. Xu., Qi-Li. Feng., Le. Kang., 2011. Cloning and expression analysis of six small heat shock protein genes in the common cutworm, Spodoptera litura. Journal of Insect Physiology. 57, 908-914. https://doi.org/10.1155/2015/132635. doi:10.1016/j.jinsphys.2011.03.026. 
Yokoyama. V.Y., Miller. G.T., Crisosto. C.H., 2001. Pest response in packed table grapes to low temperature storage combined with slow-release sulfur dioxide pads in basic and large-scale tests. J. Econ. Entomol. 4, 984-988. https://doi.org/10.1603/0022-0493-94.4.984

Yu. S.J., Liao. E.C., Tsai. J.J., 2014. House dust mite allergy: environment evaluation and disease prevention. Asia. Pac. Allergy. 4, 241-252. https://doi.org/10.5415/apallergy.2014.4.4.241 
Figures

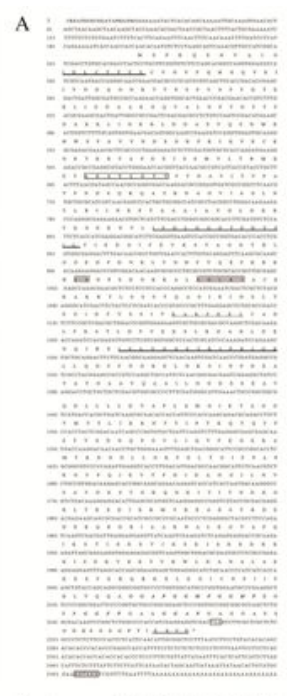

B
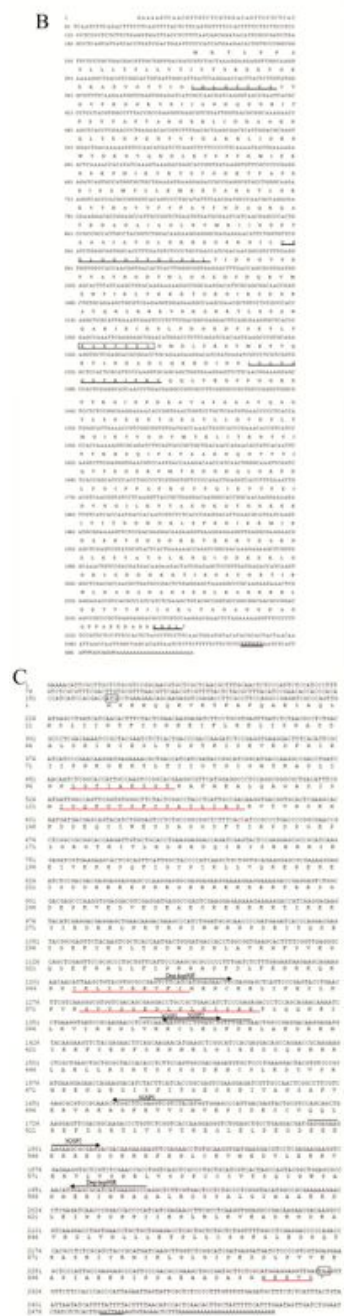

Figure 1

Nucleotide and deduced amino acid encoding region of TpHSP70-1(A)囚TpHSP70-2 (B) and TpHSP90(C). Signature sequences of TpHSP genes are marked with underline. 


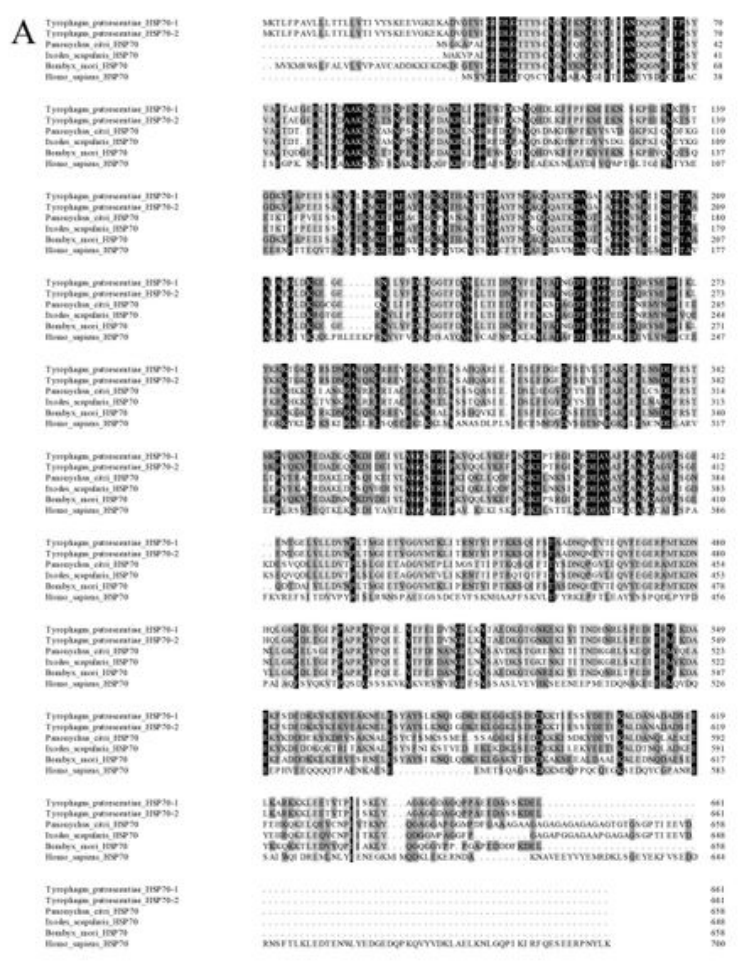

$\mathrm{B}= \pm$

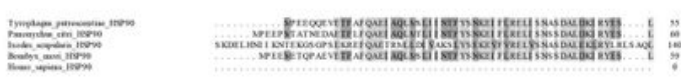

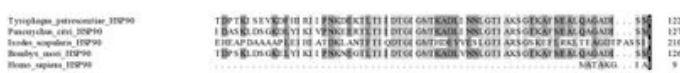
En

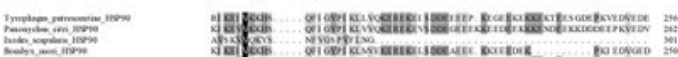
n-

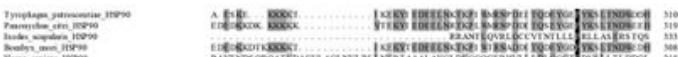

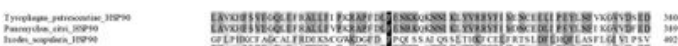

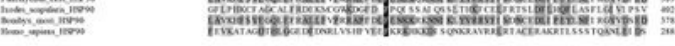

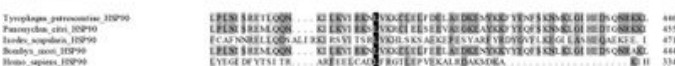

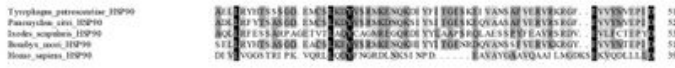

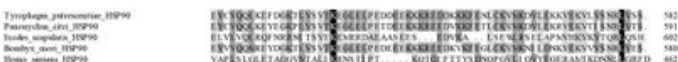

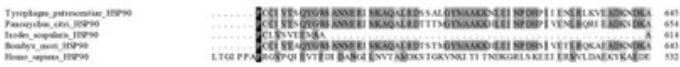

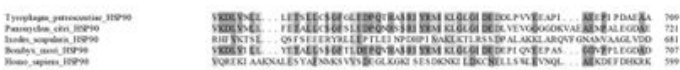

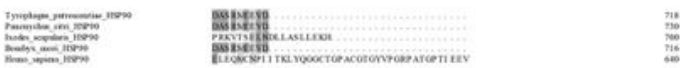

\section{Figure 2}

Comparison of the amino acid sequences of HSP70 protein family (A) and HSP90 protein family (B) 
A

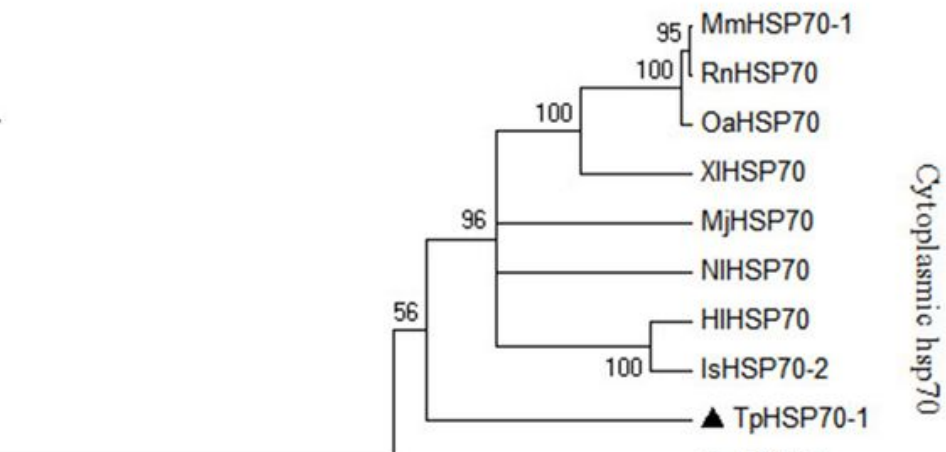

NcHSP70

100 PcHSP70-1

PcHSP70-2

TcHSP70-3

98

$100 \longleftarrow$ TuHSP70

100 [ $\mathrm{HsHSP70}$

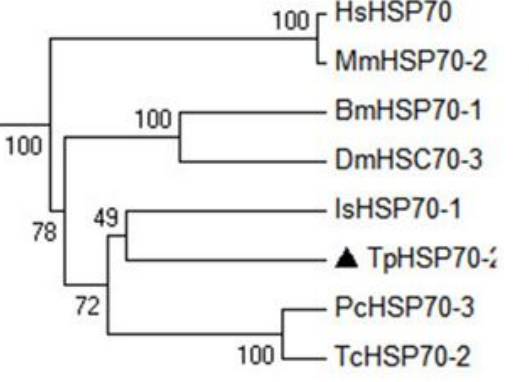

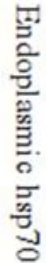

\begin{tabular}{ccccc}
1 & 1 & 1 & & \\
\hline 0.20 & 0.15 & 0.10 & 0.05 & 0.00
\end{tabular}
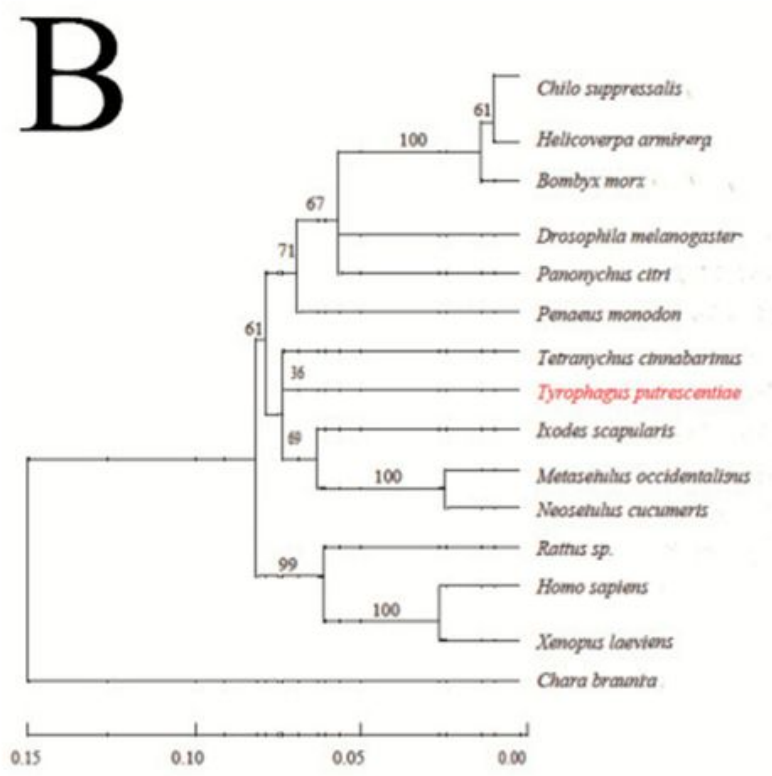

Insecta

Acari

Mammal

Amphibians

Chara

\section{Figure 3}

Phylogenetic tree of HSP70 (A) and HSP90 (B) from T. putrescentiae and other species. Constructed by the neighbor joining method based on amino acid sequences. Numbers at each branch indicate the percentage of times, and a node is supported in 1,000 bootstraps pseudo-replication by neighbor joining. 


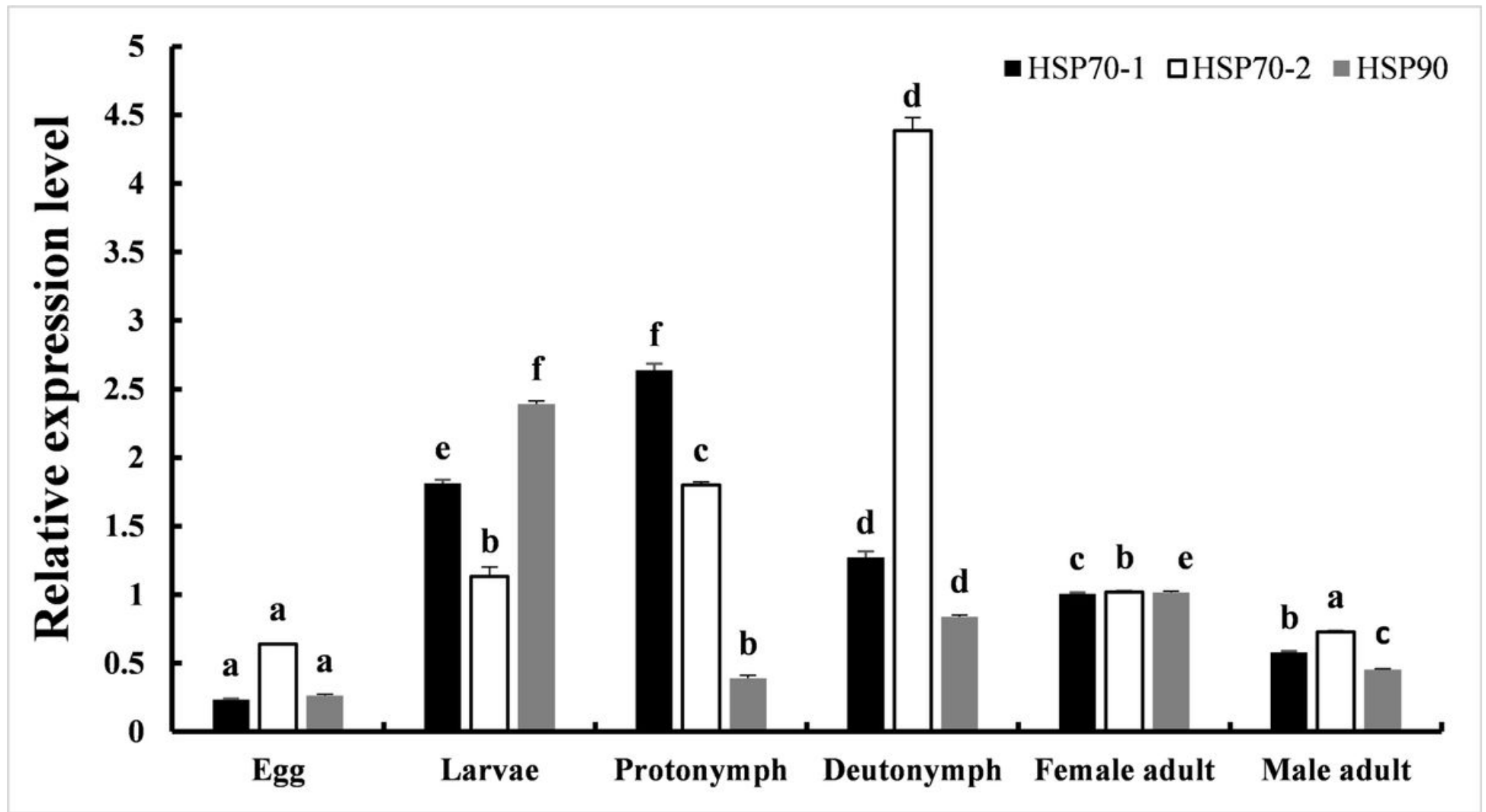

Figure 4

Stage-specific TpHSP70-1, TpHSP70-2 and TpHSP90 expression in T.putrescentiae. The mRNA expression level of TpHSP70-1, TpHSP70-2 and TpHSP90 in different developmental stages including egg, protonymph, deutonymph, tritonymph and adult stages of T.putrescentiae was measured by fluorescent real-time quantitative PCR. Values are the mean $\pm S D(n=200)$. The different letters above the bars (a-f) indicate a significant difference in the means as assessed using Duncan's multiple comparison tests $(P<0.05)$. 

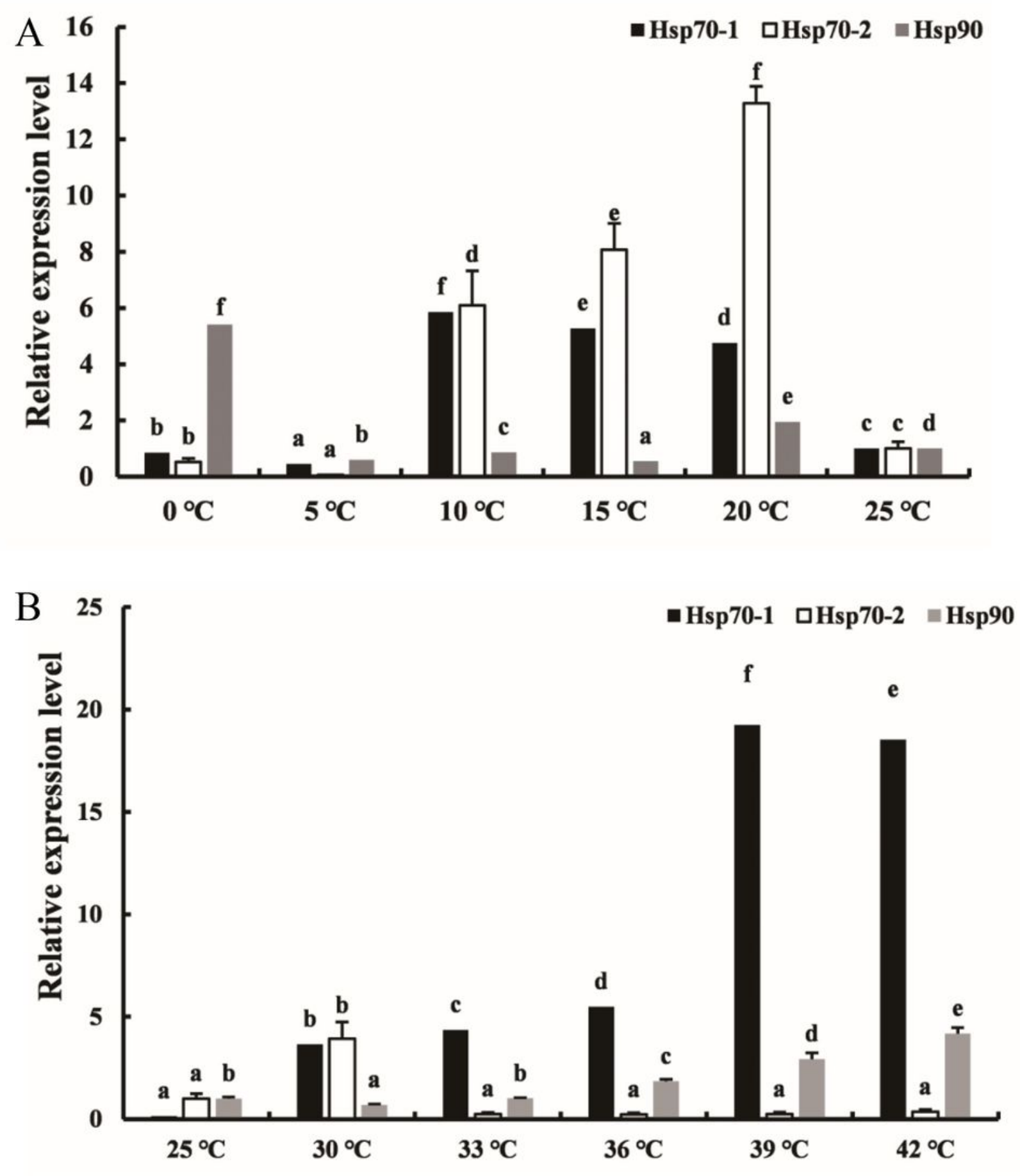

Figure 5

(A) Comparative quantitative RT-PCR analyzed of the relative expression of TpHSP 70-1, TpHSP 70-2 and

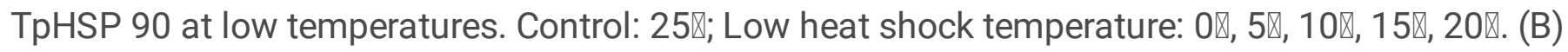
Comparative quantitative RT-PCR analyzed of the relative expression of TpHSP 70-1, TpHSP 70-2 and

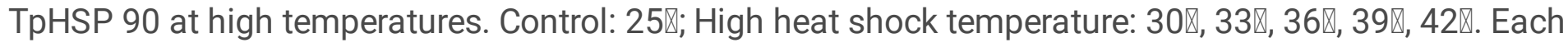
temperature treatment was three replicates. Data were represented as the mean $\pm S D(n=200)$. Letters 
above columns indicate levels of difference significance at $P<0.05$. The same letters are not significantly different, B A302 P>0.05. 4. Discussio 